\title{
Sensor Fusion-Based Vacant Parking Slot Detection and Tracking
}

\author{
Jae Kyu Suhr, Member, IEEE, and Ho Gi Jung, Senior Member, IEEE
}

\begin{abstract}
This paper proposes a vacant parking slot detection and tracking system that fuses the sensors of an Around View Monitor (AVM) system and an ultrasonic sensor-based automatic parking system. The proposed system consists of three stages: parking slot marking detection, parking slot occupancy classification, and parking slot marking tracking. The parking slot marking detection stage recognizes various types of parking slot markings using AVM image sequences. It detects parking slots in individual AVM images by exploiting a hierarchical tree structure of parking slot markings and combines sequential detection results. The parking slot occupancy classification stage identifies vacancies of detected parking slots using ultrasonic sensor data. Parking slot occupancy is probabilistically calculated by treating each parking slot region as a single cell of the occupancy grid. The parking slot marking tracking stage continuously estimates the position of the selected parking slot while the ego-vehicle is moving into it. During tracking, AVM images and motion sensor-based odometry are fused together in the chamfer score level to achieve robustness against inevitable occlusions caused by the ego-vehicle. In the experiments, it is shown that the proposed method can recognize the positions and occupancies of various types of parking slot markings and stably track them under practical situations in a real-time manner. The proposed system is expected to help drivers conveniently select one of the available parking slots and support the parking control system by continuously updating the designated target positions.
\end{abstract}

Index Terms-Around View Monitor (AVM), in-vehicle motion sensor, occupancy classification, parking assist system, parking slot marking detection and tracking, ultrasonic sensor.

\section{INTRODUCTION}

D UE TO THE rapidly growing interest in parking aid products, automatic parking systems have been extensively researched. Target position designation is one of the primary components of automatic parking systems. This has been explored in a variety of ways that can be categorized into four types: user interface-based [1]-[4], free space-based [5]-[25], parking slot marking-based [26]-[31], and infrastructure-based [32]-[39] approaches. Most of the (semi-) automatic parking system products on the market designate target positions by utilizing a user interface-based approach via a touch screen [3] or a free space-based approach via ultrasonic sensors (usually

Manuscript received April 11, 2013; revised June 19, 2013; accepted June 19, 2013. Date of publication August 8, 2013; date of current version January 31, 2014. This work was supported by the Hyundai Motor Company. The Associate Editor for this paper was A. Amditis.

J. K. Suhr is with the Research Institute of Automotive Electronics and Control, Hanyang University, Seoul 133-791, Korea.

H. G. Jung is with the Department of Automotive Engineering, Hanyang University, Seoul 133-791, Korea (e-mail: hogijung@ @anyang.ac.kr).

Color versions of one or more of the figures in this paper are available online at http://ieeexplore.ieee.org.

Digital Object Identifier 10.1109/TITS.2013.2272100

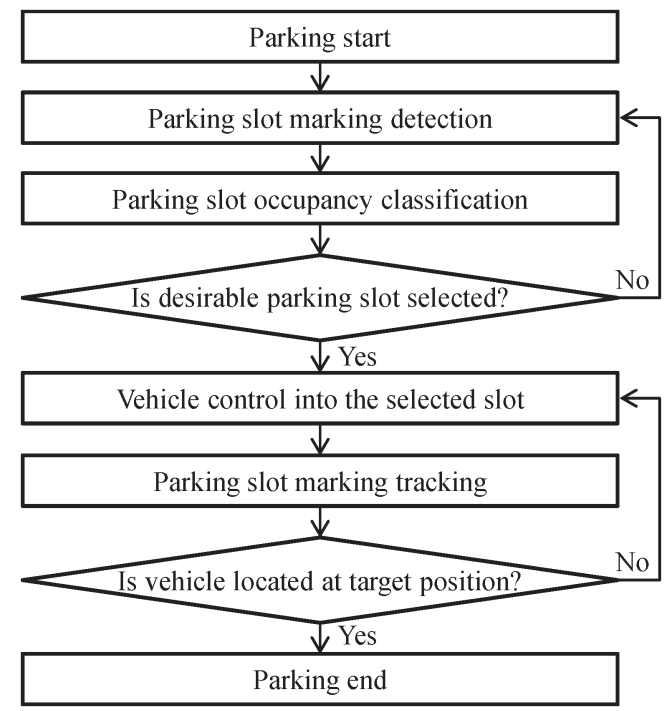

Fig. 1. Flowchart of the proposed system.

mounted on both sides of the front bumper) [9]-[12]. Once the target position is designated, the system generates a path from the initial position to the target position and autonomously controls the steering to follow the path. For this purpose, it continuously estimates the ego-vehicle position using invehicle motion sensor-based odometry. Meanwhile, an Around View Monitor (AVM) system has become popular as a parking aid product, and most car makers have started to produce vehicles equipped with this system [40]-[43]. An AVM system produces a bird's-eye view image for the $360^{\circ}$ surroundings of the vehicle by stitching together a number of images acquired by three or four cameras. Displaying AVM images helps drivers easily recognize parking slot markings and obstacles around the vehicle during the parking maneuver.

This paper proposes a vacant parking slot detection and tracking system that fuses the sensors of an AVM system and an ultrasonic sensor-based automatic parking system. The flowchart of the proposed system is presented in Fig. 1. Once a driver starts parking, the system continuously detects parking slot markings and classifies their occupancies. Simultaneously, it presents the detection and classification results on AVM images to help the driver identify available parking slots. If a driver selects a desirable parking slot using the touch screen interface, this system tracks the position of the selected parking slot while the ego-vehicle is moving into it. Finally, the parking maneuver is finished when the vehicle is located at the target position. This paper excludes path planning and steering control because they are beyond its scope. 
The proposed system consists of three stages: parking slot marking detection, parking slot occupancy classification, and parking slot marking tracking. First, the parking slot marking detection stage recognizes various types of parking slot markings in AVM image sequences. Parking slot markings are detected by a hierarchical tree structure-based method [31] in individual AVM images, and the sequential detection results are combined by using an image registration technique and slot properness measures. Second, the parking slot occupancy classification stage probabilistically identifies vacancies of the detected parking slots using ultrasonic sensor data. Occupancy classification can be modeled as a binary estimation problem with a static state if each parking slot region is treated as a single cell of the occupancy grid. The probability of parking slot occupancy is calculated utilizing ultrasonic sensor data acquired while the vehicle is passing by parking slots. Finally, the parking slot marking tracking stage continuously estimates the position of the selected parking slot while the ego-vehicle is moving into it. Directional chamfer matching (DCM) is adopted to track parking slot markings consisting of multiple lines with different orientations. During tracking, AVM images and motion sensor-based odometry are fused together in the chamfer score level to achieve robustness against inevitable occlusions caused by the ego-vehicle. In the experiments, the proposed system was tested in various practical situations, and it successfully detected and tracked vacant parking slots in a real-time manner.

The proposed system has the following advantages: 1) Unlike the free space-based approach using ultrasonic sensors, this system can more accurately detect parking spaces because its performance does not depend on the existence and poses of adjacent vehicles. 2) Compared with the user interface-based approach via a touch screen, this system is more convenient since it requires no repetitive driver operations, and drivers only need to select one of the detected parking slots. 3) The egovehicle can be more accurately settled at the target position because this system continuously estimates the position of the selected parking slot using AVM images rather than predicting the position wholly based on error-prone odometry. This system has limitations as it can be used only in cases where parking slot markings are presented. However, it is still useful since drivers need automatic parking systems generally in complex urban situations where parking slot markings are usually presented. If it is found that parking slot markings are not presented, the system can switch the target position designation method into the free space-based approach using ultrasonic sensors. This will be explained further in Section VI-F.

This paper has the following contributions:

1) It presents a novel system that fuses the sensors of an AVM system and an ultrasonic sensor-based automatic parking system and shows that the proposed system can detect and track parking slot markings more effectively than individual systems.

2) It suggests a reliable parking slot marking detection method that first detects various parking slot markings via a hierarchical tree structure-based method [31] and combines sequential detection results using an image registration technique and slot properness measures. This part is an enhancement of our previous work [44].

3) It proposes an efficient parking slot occupancy classification method that probabilistically identifies occupancies of detected parking slots based on ultrasonic sensor data by treating each parking slot region as a single cell of the occupancy grid.

4) It presents a parking slot marking tracking method that is robust against severe occlusions caused by the egovehicle. This method adopts the DCM technique and fuses AVM images and motion sensor-based odometry in the chamfer score level.

The rest of this paper is organized as follows. Section II explains related research. Sections III-V describe the stages for parking slot marking detection, parking slot occupancy classification, and parking slot marking tracking, respectively. Section VI presents experimental results and discussions. Finally, this paper is concluded with a summary in Section VII.

\section{RELATED RESEARCH}

Target position designation methods for automatic parking systems can be categorized into four types: user interface-based [1]-[4], free space-based [5]-[25], parking slot marking-based [26]-[31], and infrastructure-based [32]-[39] approaches.

\section{A. User Interface-Based Approach}

The user interface-based approach helps a driver manually select a parking space or drive into it. Although it has a drawback of repetitive driver operations, it is useful as a backup tool for failure cases of automatic methods and is easy to implement. Hiramatsu et al. [1] proposed an interactive method with steering maneuver and augmented display. This method helps a driver locate a displayed vertical pole at the end of the parked vehicle for easier parallel parking. Furutani et al. [2] presented an arrow button-based graphical user interface for target position designation. A driver repetitively clicks arrow buttons to locate a rectangle at the desired parking space. This method has been commercialized in the Toyota Prius [3]. Jung et al. [4] proposed a manual designation method based on the drag-and-drop technique. Target positions are located by dragging inside and outside of a rectangular parking slot displayed in a touch screen.

\section{B. Free Space-Based Approach}

The free space-based approach designates a target position by recognizing a vacant space between adjacent vehicles. This is the most widely used approach since adjacent vehicles can be easily recognized by various range-finding sensors. However, its performance depends on the existence and poses of adjacent vehicles. A variety of sensors have been utilized, and the ultrasonic sensor-based method is the most popular [5]-[8] among them. Usually, two ultrasonic sensors are mounted on both sides of the front bumper. Free spaces and adjacent vehicles are recognized by registering ultrasonic sensor data via invehicle motion sensors. Most car makers are manufacturing this 
method [9]-[12] because ultrasonic sensors are inexpensive and easy to install. However, this method fails in situations where there is no adjacent vehicle and in slanted parking situations where adjacent vehicle surfaces are not perpendicular to the heading directions of ultrasonic sensors. Imaging sensors have also been widely used in this approach. Adjacent vehicles are recognized by detecting horizontal edges [13] or reconstructing 3-D structures via monocular motion stereo [14]-[16], binocular stereo [17], [18], and structured light [19]. Although imaging sensor-based methods have an advantage in that existing vehicle-mounted cameras can be used, they are sensitive to illumination conditions. Abad et al. developed a multisensorbased method [20] that combines ultrasonic sensor data and rearview camera images. In addition to these sensors, laser scanners [21], [22], short-range radars [23], [24], and photonic mixer devices [25] have been utilized to recognize free spaces and adjacent vehicles.

\section{Parking Slot Marking-Based Approach}

The parking slot marking-based approach designates a target position by recognizing markings on a road surface. Its designation process does not depend on the existence and poses of adjacent vehicles, and it can simply use existing vehiclemounted cameras. However, this approach can only be used in cases where parking slot markings are presented. Jung et al. [26] proposed a one-touch type method that recognizes the line segments of parking slot markings based on a point inside the parking slot marked by a driver. Since this method can deal with only a single type of parking slot marking, they proposed a two-touch type method [27]. This method recognizes various types of parking slot markings based on two points of a parking slot entrance provided by a driver. Meanwhile, methods that do not require manual inputs have been also developed. Xu et al. [28] proposed a method that uses a neural network-based color segmentation technique to recognize pink-colored parking slot markings. Jung et al. [29] assumed that parking slot markings consist of lines with a fixed width and recognized them by applying a specialized filter to a Hough transformed bird'seye view edge image. Tanaka et al. [30] proposed a method that recognizes parking slot markings by detecting straight lines using an improved random sample consensus algorithm. Since all these automatic methods can handle only one or two types of parking slot markings, Suhr and Jung [31] proposed a fullautomatic method that is able to recognize various types of parking slot markings based on a hierarchical tree structure.

\section{Infrastructure-Based Approach}

Unlike the other approaches, the infrastructure-based approach designates a target position by utilizing devices outside the ego-vehicle via vehicle-to-infrastructure communication. The most popular system in this approach provides drivers with locations of vacant parking spaces to help them easily find or reserve parking spaces [32], [33]. Methods that actively participate in parking maneuvers have been also developed. Suzuki et al. [34] proposed a system that offers bird's-eye view images of a parking situation, including parking slot markings and adjacent vehicles using surveillance cameras. Wada et al.

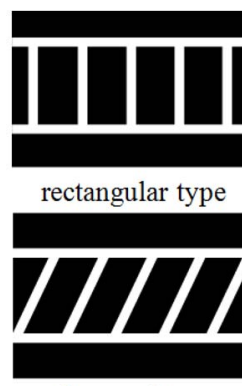

diamond type

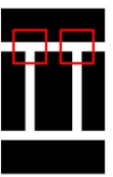

TT-slot

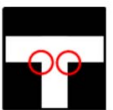

T-junction

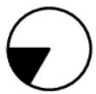

$60^{\circ}$-corner

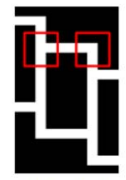

TL-slot

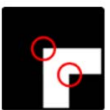

L-junction

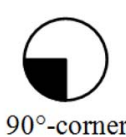

(a)

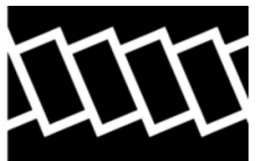

slanted rectangular type

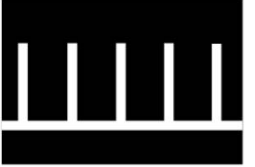

open rectangular type

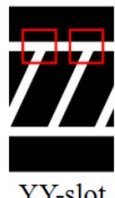

(b)

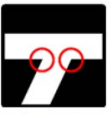

(c)

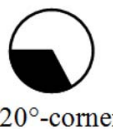

(d)

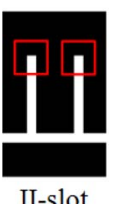

II-slot

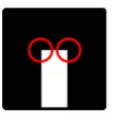

I-junction

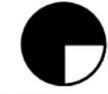

$270^{\circ}$-corner
Fig. 2. Hierarchical tree structure of parking slot markings. (a) Parking slot markings. (b) Slots. (c) Junctions. (d) Corners.

[35], [36] presented a system that assigns a target position to a vehicle entering a parking lot and provides rough guidance to the parking space entrance and fine guidance to the target position. An et al. [37] and Sung et al. [38] suggested an automatic valet parking system that conducts fully automatic parking after drivers step down from vehicles at the entrance of a building by utilizing both infrastructure and in-vehicle sensors. An automatic valet parking system has been also researched as a European Union project entitled V-Charge [39].

\section{PARKING Slot MARKING Detection}

\section{A. Hierarchical Tree Structure-Based Method}

The proposed parking slot marking detection method is based on our previous work [31] that recognizes various types of parking slot markings by modeling them as a hierarchical tree structure of a corner-junction-slot. This section briefly reviews this hierarchical tree structure-based method. The proposed system focuses on four types of parking slot markings in Korea (rectangular, slanted rectangular, diamond, and open rectangular types), as shown in Fig. 2(a). These four types of parking slot markings consist of four types of slots (TT-slot, TL-slot, YY-slot, and II-slot), as shown in Fig. 2(b), and each slot is composed of two junctions, as shown in Fig. 2(b) with red rectangles. Junctions composed of four types of slots can be categorized into four types (T-junction, L-junction, Y-junction, and I-junction), as shown in Fig. 2(c), and each junction consists of two corners, as shown in Fig. 2(c) with red circles. These corners can be divided into four types 


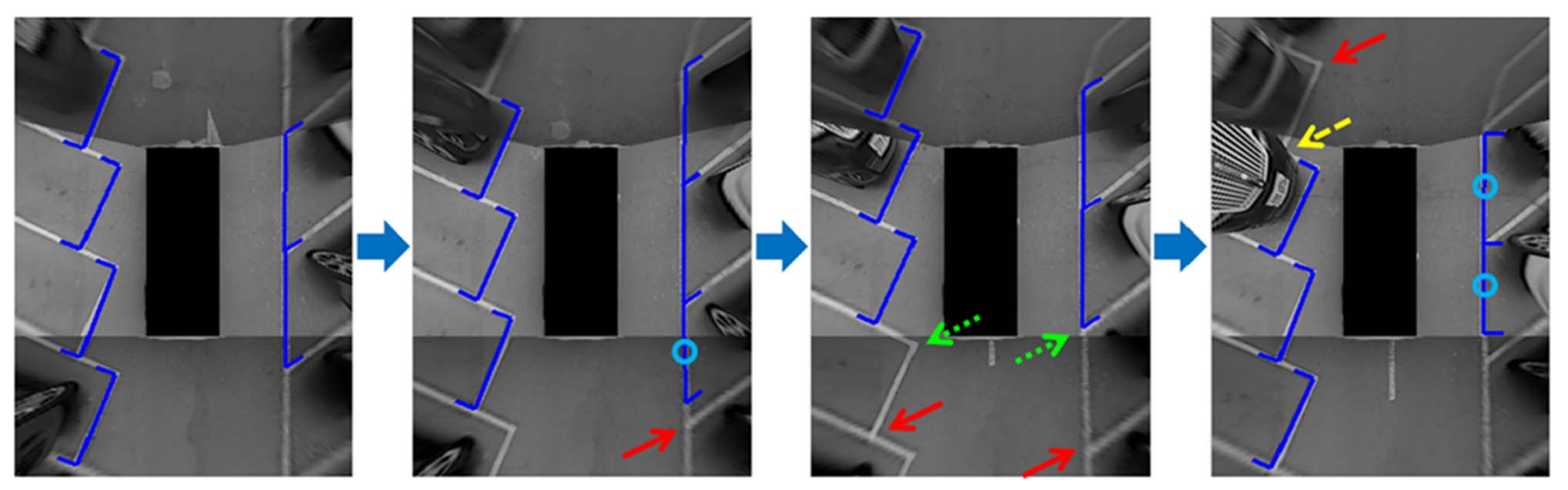

Fig. 3. Parking slot marking detection results of the hierarchical tree-structure-based method in [31].

$\left(60^{\circ}\right.$-corner, $90^{\circ}$-corner, $120^{\circ}$-corner, and $270^{\circ}$-corner) according to the arc angles of the ground plane, as shown in Fig. 2(d). This is called a hierarchical tree structure of parking slot markings.

Based on the hierarchical tree structure of a corner-junctionslot, the method in [31] first detects corners via the Harris corner detector [45] and then generates junctions by combining these corners. Finally, slots are produced from junction pairs. Junction and slot generation procedures are conducted based on the type, orientation, and position properties of corners and junctions, respectively. Since this method utilizes an approach that first produces multiple hypotheses so as not to lose the correct ones and rejects false detections via geometric constraints, it is robust against partial occlusions and damage to parking slot markings. Furthermore, it is applicable to realtime systems because of its computational simplicity. This method was evaluated with numerous practical rearview camera images and showed a better performance than the previous semi-automatic [27] and full-automatic methods [29].

\section{B. Detecting Parking Slot Markings in AVM Image Sequences}

The hierarchical tree structure-based method [31] is applied to individual AVM images sequentially taken while the egovehicle is passing by parking slots. Fig. 3 shows parking slot marking detection results in sequential AVM images using the method in [31]. In this figure, it can be seen that there are several missing slots and falsely detected slots (marked with cyan circles). Since the method in [31] was originally proposed for rearview camera images, of which the view is so narrow that the number and pose of the captured parking slot is restricted, it cannot be directly applied to AVM images. As shown in Fig. 3, situations presented in AVM images are much more diverse than those of rearview images in the following aspects: 1) Various types of numerous parking slot markings can be presented. 2) Image qualities of junctions can be degraded at locations far from cameras (red solid arrows) or stitching lines (green dashed arrows). 3) Junctions can be also occluded by adjacent vehicles (yellow dashed arrow).

To overcome this problem, this paper proposes a method that efficiently combines sequentially detected parking slots. Parking slot markings that can be detected in a single AVM image are restricted. However, occluded or degraded parking

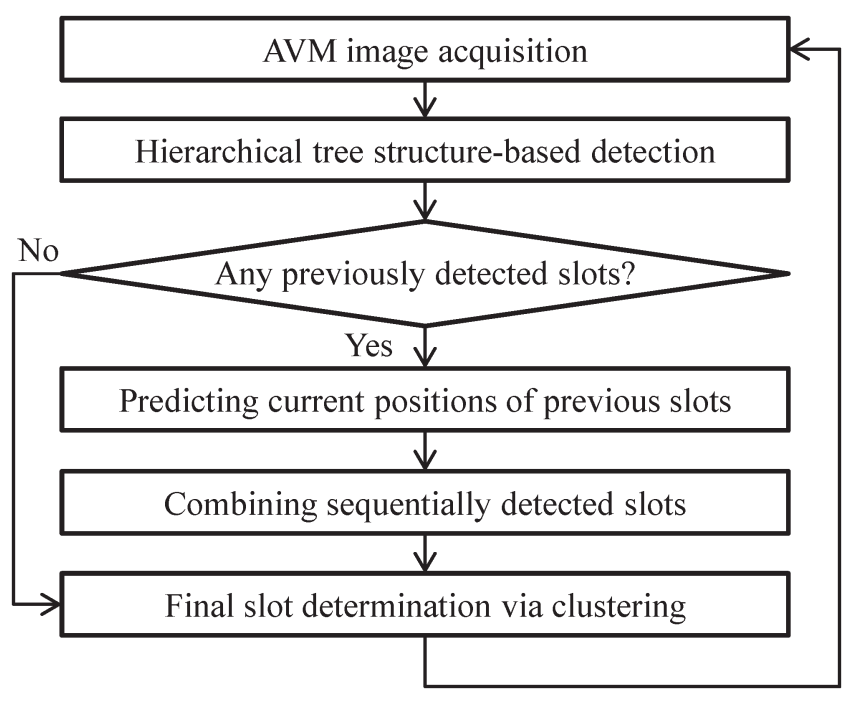

Fig. 4. Flowchart of the proposed parking slot marking detection method.

slot markings in one AVM image can be detected in other AVM images since occluded regions and image qualities are changing while the ego-vehicle is moving along the corridor of the parking lot. Therefore, it is possible to obtain more reliable detection results if sequentially detected parking slots are properly combined. Fig. 4 shows a flowchart of the proposed parking slot marking detection method. This method first detects various types of parking slot markings using the hierarchical tree structure-based method. If there are previously detected parking slots, their current positions are predicted using an image registration technique. After that, sequential detection results are combined according to slot properness measures, and final parking slots are determined via slot clustering.

\section{Predicting Current Positions of Previous Parking Slots}

To predict the current positions of previously detected parking slots, this paper utilizes an image registration technique. Transformation between sequential ground plane images is estimated, and positions of parking slots in the previous image are transformed into the current image via this transformation. Generally, if two cameras at different positions take the same ground plane, two ground plane images are related with a homography with 8 degrees of freedom [46]. Since AVM images 
are considered to be acquired by a virtual camera whose optical axis is perpendicular to the ground plane under a planar motion constraint, the relation between two ground plane images can be approximated by a similarity transformation with 3 degrees of freedom. In the case of the AVM system, images ( $\mathrm{x}$ and $\mathrm{x}^{\prime}$ ) of a 3-D point on the ground plane taken at different positions can be expressed as

$$
\begin{aligned}
& \mathbf{x}=K\left[I_{3 \times 3} \quad \mathbf{0}_{3 \times 1}\right][X, Y, H, 1]^{T} \\
& \mathbf{x}^{\prime}=K\left[R_{z, 3 \times 3} \quad \mathbf{t}_{x y, 3 \times 1}\right][X, Y, H, 1]^{T}
\end{aligned}
$$

where $K, I_{3 \times 3}$, and $0_{3 \times 1}$ are a $3 \times 3$ intrinsic parameter matrix of a virtual camera, a $3 \times 3$ identity matrix, and a $3 \times 1$ zero vector, respectively. $R_{z, 3 \times 3}$ and $\mathbf{t}_{x y, 3 \times 1}$ are a $3 \times 3$ rotation matrix about the $z$-axis and a $3 \times 1$ translation vector in the $x y$-plane, respectively. $(X, Y, H)$ are coordinates of a 3-D point, and $H$ represents the camera height from the ground plane. If (1) and (2) can be converted into (3) and (4), respectively, (5) is obtained by substituting (3) into (4).

$$
\begin{aligned}
& K^{-1} \mathbf{x}=[X, Y, H]^{T} \\
& \mathbf{x}^{\prime}=K\left[\begin{array}{cc}
R_{2 \times 2} & \mathbf{t}_{2 \times 1} / H \\
\mathbf{0}_{2 \times 1}^{T} & 1
\end{array}\right][X, Y, H]^{T} \\
& \mathbf{x}^{\prime}=K\left[\begin{array}{cc}
R_{2 \times 2} & \mathbf{t}_{2 \times 1} / H \\
\mathbf{0}_{2 \times 1}^{T} & 1
\end{array}\right] K^{-1} \mathbf{x}
\end{aligned}
$$

where $R_{2 \times 2}, \mathbf{t}_{2 \times 1}$, and $\mathbf{0}_{2 \times 1}$ are a $2 \times 2$ rotation matrix, a $2 \times 1$ translation vector, and a $2 \times 1$ zero vector, respectively. If (5) is expressed in inhomogeneous coordinates, (6) is produced.

$$
\begin{aligned}
& \mathbf{x}_{\text {in }}^{\prime}= R_{2 \times 2} \mathbf{x}_{\text {in }}+\mathbf{t} \\
& \quad \text { where } \quad \mathbf{t}=f \cdot \mathbf{t}_{2 \times 1} / H+\mathbf{o}-R_{2 \times 2} \mathbf{o}
\end{aligned}
$$

where $\mathbf{x}_{\text {in }}$ and $\mathbf{x}_{\text {in }}^{\prime}$ are $\mathbf{x}$ and $\mathbf{x}^{\prime}$ in inhomogeneous coordinates, respectively. $f$ and $\mathbf{o}$ are the focal length and the principal point of a virtual camera, respectively. Since the ground plane regions in two AVM images are related with (6), it is possible to predict the current positions of previously detected parking slots using three parameters. These parameters can be estimated via Procrustes analysis [47] in the following equations:

$$
\begin{aligned}
t_{x}= & \frac{1}{N_{P}}\left\{\sum_{i=1}^{N_{P}}\left(x_{i}^{\prime}-x_{i}\right)\right\} \\
t_{y}= & \frac{1}{N_{P}}\left\{\sum_{i=1}^{N_{P}}\left(y_{i}^{\prime}-y_{i}\right)\right\} \\
\theta & =\tan ^{-1}\left\{\sum_{i=1}^{N_{P}}\left(\bar{x}_{i} y_{i}-\bar{y}_{i} x_{i}\right) / \sum_{i=1}^{N_{P}}\left(\bar{x}_{i} x_{i}+\bar{y}_{i} y_{i}\right)\right\} \\
& \text { where } \quad \bar{x}_{i}=x_{i}^{\prime}-t_{x} \text { and } \quad \bar{y}_{i}=y_{i}^{\prime}-t_{y}
\end{aligned}
$$

where $N_{P}$ indicates the number of corresponding feature points, and $\left(x_{i}, y_{i}\right)$ and $\left(x_{i}^{\prime}, y_{i}^{\prime}\right)$ are the positions of the corresponding feature points in the previous and current images, respectively. This paper reuses the corners detected by the Harris corner detector in the parking slot marking detection procedure as feature points and matches them via normalized cross correlation [48]. Reuse of the corners can save computational costs. Since outliers are inevitably included during the feature point matching process, final parameters are estimated by the random sample consensus (RANSAC) method [49], which is one of the most widely used robust estimators. Inlier corners classified by RANSAC are more stable than the others since they are continuously detected and proved to be on the ground plane. Thus, only the inlier corners are utilized to generate junctions and slots during the parking slot marking detection procedure. This approach reduces both computational cost and false detections.

\section{Combining Sequentially Detected Parking Slots}

Once positions of parking slots are detected and predicted, these sequentially detected parking slots are combined to produce more reliable results. For this task, overlapping situations of the detected and predicted parking slots are first checked by using the Jaccard coefficient [50] in the following equation:

$$
J\left(S_{i}, S_{j}\right)=\frac{\left|S_{i} \cap S_{j}\right|}{\left|S_{i} \cup S_{j}\right|}
$$

where $J\left(S_{i}, S_{j}\right)$ indicates the Jaccard coefficient between two rectangles formed by the $i$ th slot $\left(S_{i}\right)$ and the $j$ th slot $\left(S_{j}\right)$. Overlapping situations are classified into the following three cases according to the Jaccard coefficient and two predetermined thresholds, i.e., $T_{1}$ and $T_{2}\left(T_{1}<T_{2}\right)$.

Case I $J\left(S_{i}, S_{j}\right)<T_{1}$ : If the Jaccard coefficient between the $i$ th slot $\left(S_{i}\right)$ detected in the current image and the $j$ th slot $\left(S_{j}\right)$ predicted from a previous image is less than $T_{1}$, two slots are considered to be nonoverlapping. If $S_{i}$ does not overlap with any previously detected slots, $S_{i}$ is identified as a newly detected slot.

Case II $J\left(S_{i}, S_{j}\right) \geq T_{2}$ : If the Jaccard coefficient is greater than or equal to $T_{2}$, it can be considered that the same slot is repetitively detected in sequential images. In this case, the position of this parking slot is corrected by selecting one of two slot positions, which gives a larger normalized average intensity value $(N A I V)$ than the other. $N A I V$ is calculated as

$$
N A I V_{i}=\frac{1}{M A X(I)}\left\{\frac{1}{N_{S_{i}}} \sum_{(x, y) \subset S_{i}} I(x, y)\right\}
$$

where $M A X(I)$ is a maximum intensity value of image $I$, and $N_{S_{i}}$ and $(x, y)$ are the number of pixels in the skeleton of the parking slot entrance and their locations in the $x$ and $y$ axes, respectively. Since parking slot markings are much brighter than the ground plane and pixels near the center of the parking slot marking lines tend to have larger intensity values than the boundary pixels [26], the parking slot located at the most appropriate position has the largest $N A I V$ compared with the others. The number of repeated detections $\left(M_{i}\right)$ is also counted for this parking slot.

Case III $T_{1} \leq J\left(S_{i}, S_{j}\right)<T_{2}$ : If the Jaccard coefficient is between $T_{1}$ and $T_{2}$, two slots are considered to be overlapping, but they are not a repetitive detection of the same slot. Since two different parking slots are unable to overlap 

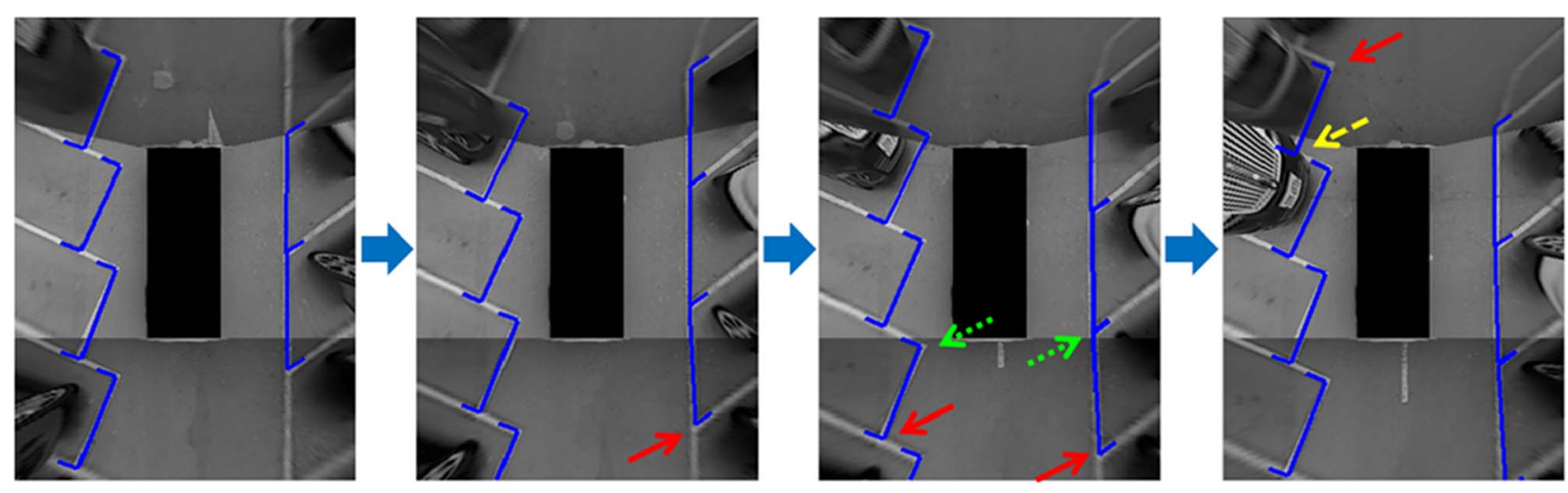

Fig. 5. Parking slot marking detection results of the proposed method.

in real situations, only one of the two slots should be selected. Two slot properness measures are utilized for this task. One is $N A I V$ in (11), and the other is normalized repeated detection count $(N R D C) . N R D C$ is calculated by normalizing the number of repeated detections $\left(M_{i}\right)$ counted in Case II, as follows:

$$
N R D C_{i}=\min \left\{\frac{M_{i}}{M_{\max }}, 1\right\}
$$

where $M_{\max }$ is the maximum repeated detection number, and it is experimentally set to $10 . N R D C$ can supplement the fault of $N A I V$ because $N A I V$ of the falsely detected slot might be larger than that of the correctly detected slot due to occlusion and illumination variations. Since $N A I V$ and $N R D C$ are normalized to a value from 0 to 1 , they are fused by the sum rule [51]. In other words, the slot that has a larger summation of $N A I V$ and $N R D C$ survives in Case III. If one would like to consider that two slots do not overlap each other in the case of overlapping of less than $10 \%, T_{1}$ can be set to $10 /(100+100-10)=0.053$ by assuming that two slot areas are the same (100). In the same way, $T_{2}$ can be set to $90 /(100+100-90)=0.818$ if one would like to consider that two slots are repetitive detections of the same slot in the case of overlapping of more than $90 \%$.

\section{E. Determining Final Parking Slots}

After combining sequentially detected parking slots, resulting slots are clustered according to their type and orientation. This system only displays the clusters that include more than or equal to two slots for providing more reliable detection results to drivers. The cluster with a single slot is not displayed but is internally stored waiting for more slots that will be included in this cluster to be displayed. Fig. 5 shows the parking slot marking detection results produced by combining sequentially detected parking slots according to the proposed method. The situations presented in this figure are exactly the same as those in Fig. 3. In Figs. 3 and 5, it can be easily noticed that the proposed method more reliably detects parking slot markings in sequential AVM images compared with the previous method [31]. More results and quantitative evaluation will be presented in Section VI.

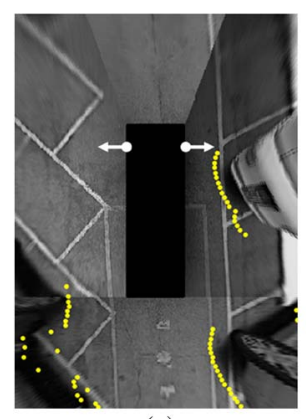

(a)

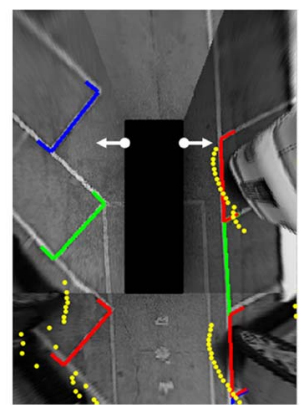

(b)
Fig. 6. (a) Ultrasonic sensor data aligned with an AVM image. (b) Parking slot occupancy classification result.

\section{PARking Slot Occupancy Classification}

The proposed system classifies occupancies of the detected parking slots by utilizing ultrasonic sensors mounted on both sides of the front bumper. For efficient classification, an occupancy grid approach is adopted. Generally, the occupancy grid approach divides an interesting area into grids and calculates the posterior probability for each cell to be occupied using the given sensor data [52]. However, the proposed system is not concerned with detailed obstacle shapes around the vehicle but with occupancy of each parking slot. Thus, this paper treats each parking slot region as a single cell of the occupancy grid and calculates the posterior probability for each slot to be occupied. This modification enhances the efficiency of parking slot occupancy classification since it avoids noninteresting regions to be involved in the calculation and handles only a small number of large-sized cells rather than numerous small-sized ones. A parking slot region has a rectangular or a diamond shape depending on its type, as shown in Fig. 2(b).

Since locations of ultrasonic sensors are static in AVM images and the relation between a pixel and physical distance is known from the calibration process, ultrasonic sensor data can be easily aligned with AVM images. Yellow dots in Fig. 6(a) show ultrasonic sensor data aligned with an AVM image, and white arrows indicate locations and heading directions of the ultrasonic sensors. If each parking slot region is treated as a cell of the occupancy grid, occupancy of each parking slot can be modeled as a binary estimation problem with a static state [52]. The posterior probability of parking slot occupancy can be sequentially calculated in log odds representation based on 
ultrasonic sensor data acquired while the ego-vehicle is passing by parking slots, as follows:

$$
\begin{aligned}
l_{1: t}(O) & =\log \frac{p\left(O \mid z_{1: t}\right)}{1-p\left(O \mid z_{1: t}\right)} \\
& =\log \left\{\frac{p\left(O \mid z_{t}\right)}{1-p\left(O \mid z_{t}\right)} \cdot \frac{p\left(O \mid z_{1: t-1}\right)}{1-p\left(O \mid z_{1: t-1}\right)} \cdot \frac{1-p(O)}{p(O)}\right\} \\
& =\log \frac{p\left(O \mid z_{t}\right)}{1-p\left(O \mid z_{t}\right)}+\log \frac{p\left(O \mid z_{1: t-1}\right)}{1-p\left(O \mid z_{1: t-1}\right)}-\log \frac{p(O)}{1-p(O)} \\
& =l_{t}(O)+l_{1: t-1}(O)-l_{o}(O)
\end{aligned}
$$

where $O$ means the parking slot is occupied, whereas $V$ means the parking slot is vacant. $z_{t}$ is a binary variable that represents whether the ultrasonic sensor data of time index $t$ is located inside a specific parking slot region. $z_{t}$ is either positive $(P)$ or negative $(N) \cdot p\left(O \mid z_{1: t}\right)$ denotes a posterior probability for the parking slot to be occupied given the ultrasonic sensor data acquired from time 1 to $t$, and $p(O)$ is a prior probability for the parking slot to be occupied. Since $p\left(O \mid z_{t}\right)$ consists of $p(O \mid P)$ and $p(O \mid N), p(O \mid P)$ and $p(O \mid N)$ are required to utilize (13). By Bayes' theorem, they are calculated from $p(P \mid O)$ and $p(P \mid V)$, which are estimated with training samples. Details of this procedure will be explained in Section VI. The posterior probability is easily recovered from the log odds ratio via

$$
p\left(O \mid z_{1: t}\right)=1-\frac{1}{1+\exp \left\{l_{1: t}(O)\right\}} .
$$

Finally, occupancies of the detected parking slots are classified according to the calculated posterior probabilities. Since ultrasonic sensor data can be measured outside of the parking slots, parking slot regions used for occupancy classification are set to be slightly larger than the actual ones by $1.5 \mathrm{~m}$ in a longitudinal direction. Fig. 6(b) shows an occupancy classification result. Parking slots with green and red lines indicate vacant and occupied parking slots, respectively, and a blue line indicates an unclassified parking slot where ultrasonic sensors have not yet scanned. More results, a detailed training process, and quantitative evaluation will be presented in Section VI.

\section{PARKing Slot Marking Tracking}

If a parking slot is detected in an AVM image, its 3-D position can be reconstructed using camera parameters estimated from the calibration procedure. However, if the assumptions of the AVM system, that is, the fixed camera configuration (height and angle) and flat road surface, are broken, the reconstructed 3-D position will be distorted. Additionally, if a 3-D position is updated using only motion sensor-based odometry, its accuracy will be degraded by a cumulative error of odometry. To alleviate this problem, this paper continuously tracks and updates the position of the selected parking slot in sequential AVM images while the vehicle is moving into it. This approach is useful because the parking slot position in an AVM image becomes more accurate when the ego-vehicle is getting closer to it. This paper proposes a DCM-based parking slot marking tracking method that fuses AVM images and motion sensor-based odometry in the chamfer score level to achieve robustness against inevitable occlusions caused by the ego-vehicle.

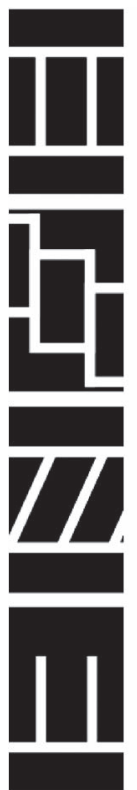

(a)

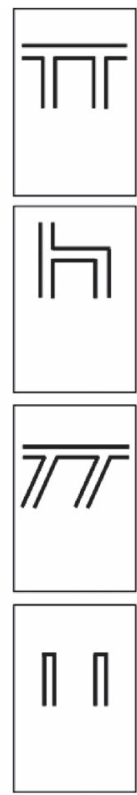

(b)

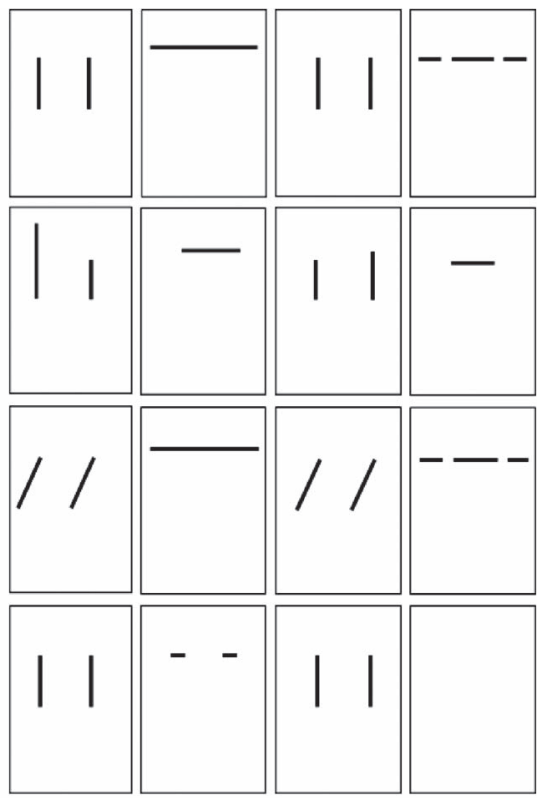

(c)
Fig. 7. Templates of various types of parking slot markings and their directional components. (a) Parking slot markings. (b) Templates. (c) Directional components of templates.

\section{A. DCM-Based Parking Slot Marking Tracking}

Parking slot markings are composed of multiple lines with different orientations, as shown in Fig. 7(a). Based on this property, this paper generates templates using multiple directional lines and estimates positions of parking slot markings using DCM in sequential AVM images. DCM has been used to overcome the distinctiveness limitation of conventional chamfer matching that equally treats all edge points. DCM used in [53] divides edge points into discrete orientation channels and calculates individual chamfer distances. These distances are combined into one to obtain a DCM score $\left(d_{\mathrm{DCM}}\right)$ as

$$
d_{\mathrm{DCM}}(\mathbf{W})=\frac{1}{N_{T}} \sum_{r=1}^{N_{D}}\left(\sum_{\mathbf{x}_{T} \in T_{r}} \min _{\mathbf{x}_{E} \in E_{r}^{\text {IMAGE }}}\left\|\mathbf{W}\left(\mathbf{x}_{T}\right)-\mathbf{x}_{E}\right\|_{2}\right)
$$

where $T_{r}$ and $E_{r}^{\mathrm{IMAGE}}$ are a template and a query edge image in the $r$ th orientation channel, respectively, and $\mathbf{x}_{T}$ and $\mathbf{x}_{E}$ are locations of edge points in $T_{r}$ and $E_{r}^{\mathrm{IMAGE}}$, respectively. W, $N_{T}$, and $N_{D}$ indicate the template transformation function, the number of edge points in the whole template $(T)$, and the number of discrete orientation channels, respectively. The DCM score can be efficiently computed via a distance transform (DT) image. DCM has more discriminatory power compared with conventional chamfer matching; but its computational cost proportionally increases with the number of orientation channels. However, this drawback is negligible in this case because parking slot markings consist of only three or four discrete orientations unlike general objects having edges of almost all orientations. Fig. 7 shows templates of various types of parking slot markings and their directional components. It can be seen that templates have, at most, four directions. As parking slot markings are assumed to be much brighter than the ground plane, both edge orientation and intensity gradient are 


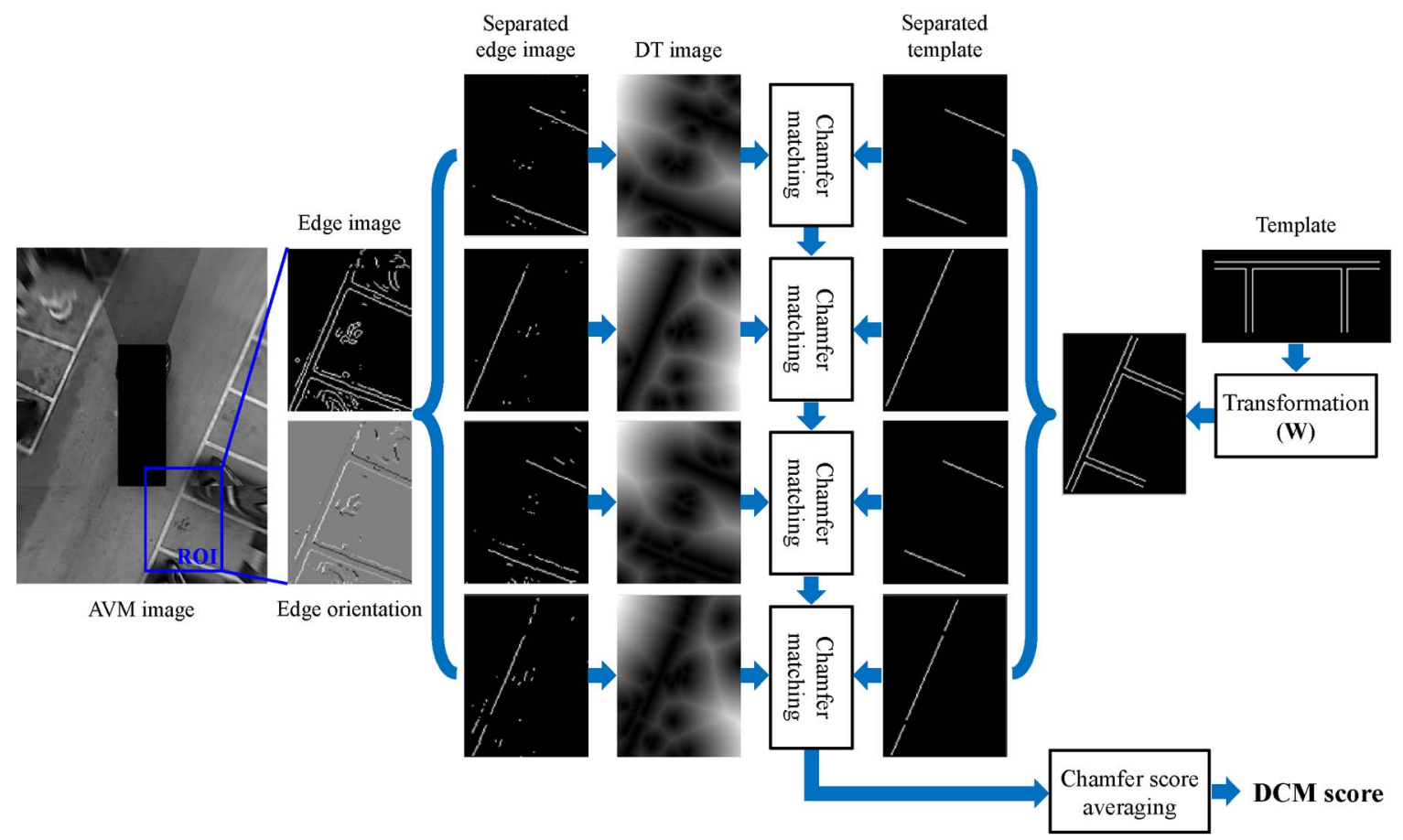

Fig. 8. Diagram for DCM-based parking slot marking tracking in case of a TT-slot.
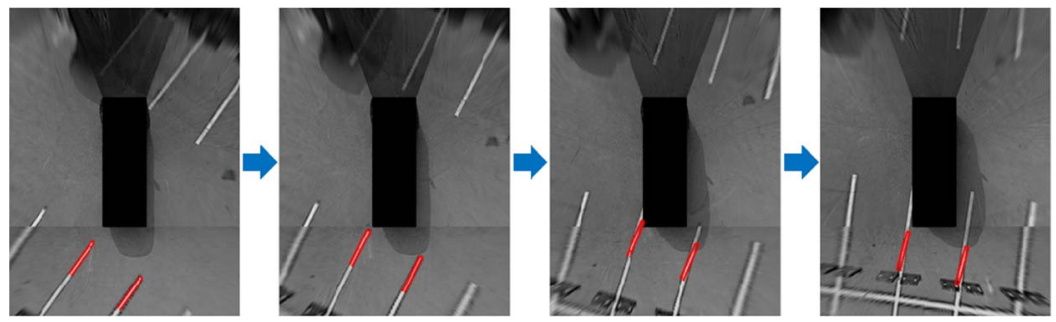

Fig. 9. Example result of DCM-based parking slot marking tracking using AVM images.

considered when separating edge points. The templates in Fig. 7 are automatically generated using the information on locations and orientations of corners and junctions that compose the selected parking slots.

To equally weight chamfer scores of all orientation channels, (15) is slightly modified into

$$
\begin{aligned}
& d_{\mathrm{DCM}}(\mathbf{W}) \\
& =\frac{1}{N_{D}} \sum_{r=1}^{N_{D}}\left\{\frac{1}{N_{T_{r}}} \sum_{\mathbf{x}_{T} \in T_{r}}\left(\min _{\mathbf{x}_{E} \in E_{r}^{\mathrm{IMAGE}}}\left\|\mathbf{W}\left(\mathbf{x}_{T}\right)-\mathbf{x}_{E}\right\|_{2}\right)\right\}
\end{aligned}
$$

where $N_{T_{r}}$ indicates the number of edge points in $T_{r}$. Fig. 8 shows a diagram for DCM-based parking slot marking tracking via (16) in the case of a TT-slot. First, the region of interest (ROI) in a query AVM image is cropped, and edge points and their orientations are calculated by the Sobel operator [48]. Basically, the ROI can be established based on the previous detection result. Then, edge points are divided into four orientation channels, and individual DT images are calculated. The template is transformed by $\mathbf{W}$ that includes changes in the 2-D location and orientation, and the transformed template is also divided into four orientation channels. Chamfer scores are indi- vidually calculated from DT images and separated templates of corresponding orientation channels. Finally, the DCM score is obtained by averaging four chamfer scores.

As it is inevitable for parking slot markings to be occluded by the ego-vehicle in parking situations, the DCM score $\left(d_{\mathrm{DCM}}\right)$ in (16) is calculated only inside the nonoccluded image regions. Since the ego-vehicle region is fixed in AVM images (a black rectangular region in the AVM image in Fig. 8), nonoccluded edge points of the template and query image can be easily determined. In addition, edge points are not generated on the boundary of the ego-vehicle region and stitching lines of four bird's-eye view images.

\section{B. Fusion of AVM Image and Odometry}

DCM-based tracking using AVM images properly works in cases where parking slot markings are slightly occluded. However, there are mainly two drawbacks in this approach because it only depends on AVM images. One drawback is that the ROI in a query AVM image should be large since this region is basically set based on the previous position of a tracked parking slot. This increases computational costs. The other drawback is that tracking performance can be affected by severe occlusion caused by the ego-vehicle. Fig. 9 shows an example situation 


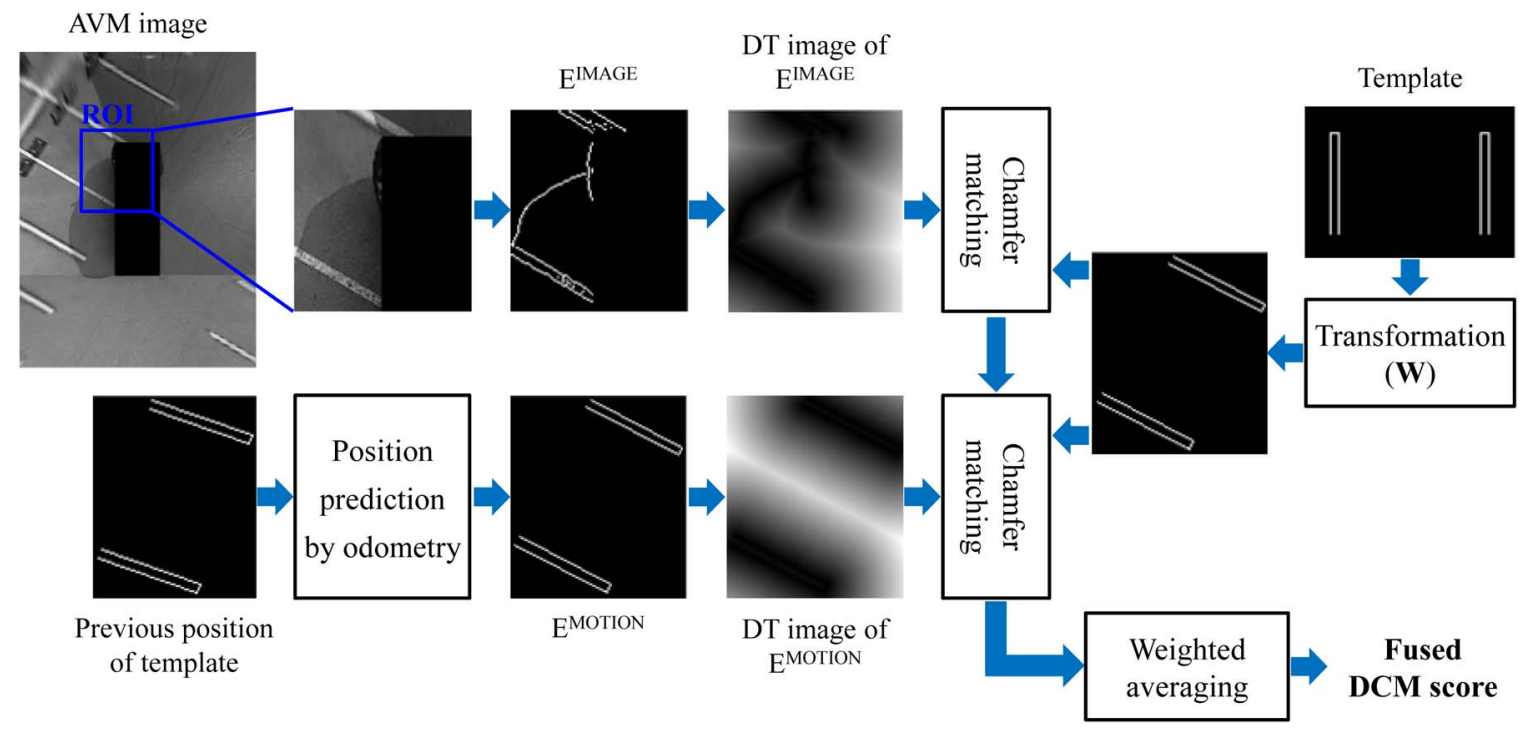

Fig. 10. Diagram for fusing an AVM image and odometry.

where II-slot is tracked by DCM-based tracking using AVM images. In this figure, red lines and a black rectangle indicate the tracked template and the ego-vehicle region, respectively. It can be seen that tracking fails when an important part of the parking slot marking is severely occluded by the ego-vehicle region. This failure frequently occurs in case of II-slot tracking since there is a small amount of latitudinal edges in this type of parking slot marking.

To overcome these drawbacks, this paper proposes a method that fuses AVM images and motion sensor-based odometry. AVM images give information independent of previous situations, but this information can be lessened due to occlusions. On the other hand, odometry gives information independent of occlusions, but it suffers from cumulative errors. Thus, parking slot markings can precisely and stably be tracked by taking advantage of these two sensors. For convenience, motion sensorbased odometry is abbreviated to odometry in this section. Odometry is estimated using wheel speed and steering angle sensors [54]. Odometry is utilized in most ultrasonic sensorbased automatic parking systems to update the ego-vehicle position. Image registration-based odometry used in the parking slot marking detection stage (see Section III-C) is not utilized in this stage because less image features are presented on the ground plane while the ego-vehicle is moving into the parking slot. This makes the registration-based odometry unstable.

In this paper, odometry is used to predict the current position of the previously estimated parking slot. This prediction result has a dual purpose. One is to limit the ROI, and the other is to achieve robustness against occlusions. For the first purpose, the $\mathrm{ROI}$ is determined in accordance with the current parking slot position predicted by odometry rather than the previous parking slot position. In experiments, changes in the 2-D location and orientation of $\mathbf{W}$ should be allowed up to \pm 15 pixels and $\pm 5^{\circ}$, respectively, if those ranges are determined according to the previous parking slot position. However, if the predicted parking slot position is utilized, those ranges can be reduced to \pm 2 pixels and $\pm 1^{\circ}$, respectively. Consequently, computational cost is reduced by avoiding the tracking algorithm from searching unnecessary ranges. Detailed results will be discussed in Section VI.

To achieve robustness against occlusions, this paper fuses an AVM image-based edge ( $\left.E^{\mathrm{IMAGE}}\right)$ and an odometry-based edge $\left(E^{\mathrm{MOTION}}\right) \cdot E^{\mathrm{MOTION}}$ is produced by locating the template edge at the predicted parking slot position. Since two edge images ( $E^{\mathrm{IMAGE}}$ and $\left.E^{\mathrm{MOTION}}\right)$ are binary, it is troublesome to fuse two binary images into one. Furthermore, fusion in the edge image level makes it difficult to adaptively weight $E^{\mathrm{IMAGE}}$ and $E^{\mathrm{MOTION}}$. Therefore, $E^{\mathrm{IMAGE}}$ and $E^{\mathrm{MOTION}}$ are fused in the DCM score level as

$$
\begin{aligned}
& d_{\mathrm{DCM}}^{\prime}(\mathbf{W}) \\
& =\frac{1}{N_{D}}\left[\sum_{r=1}^{N_{D}}\left\{\left(1-\alpha_{r}\right) \cdot \frac{1}{N_{T_{r}^{\prime}}} \sum_{\mathbf{x}_{T} \in T_{r}^{\prime}}\left(\min _{\mathbf{x}_{E} \in E_{r}^{\mathrm{IMAGE}}}\left\|\mathbf{W}\left(\mathbf{x}_{T}\right)-\mathbf{x}_{E}\right\|_{2}\right)\right\}\right. \\
& \left.\quad+\sum_{r=1}^{N_{D}}\left\{\alpha_{r} \cdot \frac{1}{N_{T_{r}}} \sum_{\mathbf{x}_{T} \in T_{r}}\left(\min _{\mathbf{x}_{E} \in E_{r}^{\mathrm{MOTION}}}\left\|\mathbf{W}\left(\mathbf{x}_{T}\right)-\mathbf{x}_{E}\right\|_{2}\right)\right\}\right]
\end{aligned}
$$

where $\alpha_{r}$ is a weight value for the $r$ th orientation channel. In (17), a DCM score of $E^{\mathrm{IMAGE}}$ is calculated with nonoccluded edge points $\left(T_{r}^{\prime}\right)$, and the DCM score of $E^{\mathrm{MOTION}}$ is calculated with all edge points $\left(T_{r}\right)$. Since DCM scores are continuous values unlike binary images, it is easy to fuse $E^{\mathrm{IMAGE}}$ and $E^{\mathrm{MOTION}}$ by adaptively changing the weight. $\alpha_{r}$ is calculated in accordance with the degree of occlusion caused by the egovehicle as

$$
\alpha_{r}=\left(1-\frac{N_{T_{r}^{\prime}}}{N_{T_{r}}}\right)^{P}
$$

where $N_{T_{r}^{\prime}}$ and $N_{T_{r}}$ are numbers of nonoccluded edge points and all edge points in the $r$ th orientation channel, respectively. $\alpha_{r}$ becomes closer to 0 when an amount of occlusion is being decreased. $P$ is experimentally tuned to 5 . Fig. 10 shows a diagram for fusing an AVM image and odometry via (17). The 


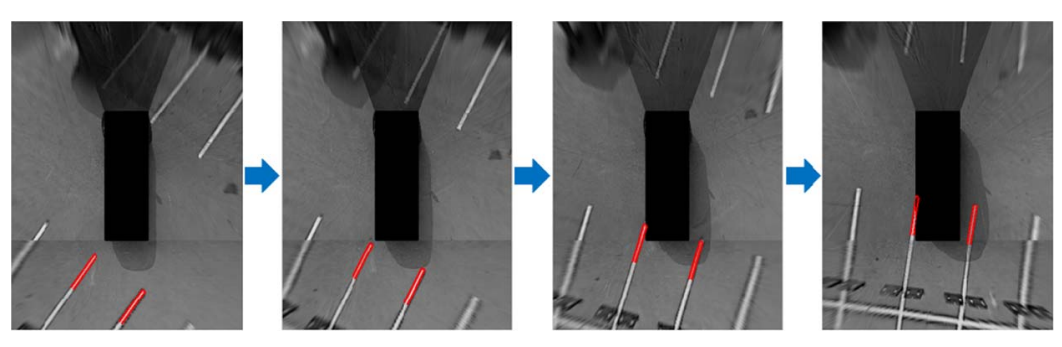

Fig. 11. Example result of the proposed fusion-based parking slot marking tracking method.

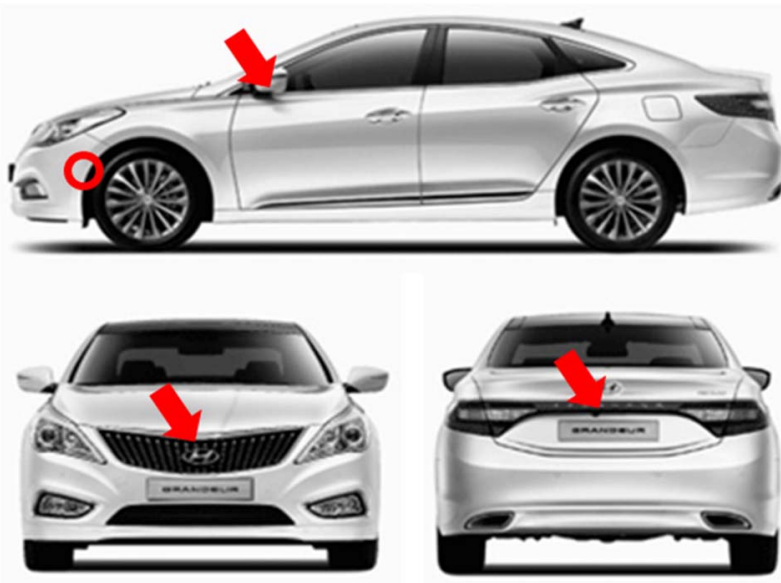

Fig. 12. Locations of ultrasonic sensors and cameras of the AVM system.

proposed method first generates $E^{\mathrm{IMAGE}}$ by detecting edges in the ROI of the AVM image and $E^{\mathrm{MOTION}}$ by predicting the current position of the template via odometry. Then, the DCM score of the AVM image is calculated using the DT image of $E^{\mathrm{IMAGE}}$ and the transformed template, and the DCM score of odometry is calculated using the DT image of $E^{\mathrm{MOTION}}$ and the transformed template. Finally, the fused DCM score is obtained by taking the weighted sum of two DCM scores. In this diagram, orientation channels are omitted for better understanding; however, this procedure is actually conducted in a DCM framework. Fig. 11 shows the tracking results obtained by fusing the AVM images and odometry. The situation presented in this figure is exactly the same as the situation in Fig. 9. It can be easily noticed that the parking slot marking is correctly tracked despite severe occlusions on its important parts. More results and quantitative evaluation will be presented in Section VI.

\section{EXPERIMENTS}

\section{A. Description of Database}

Databases used in experiments were acquired by an AVM system and an ultrasonic sensor-based automatic parking system on a Hyundai Azera [12]. Two ultrasonic sensors are mounted on both sides of the front bumper, as shown in Fig. 12 with a red circle. Their operating range, resolution, and acquisition frequency are 30-450 cm, $2 \mathrm{~cm}$, and $15 \mathrm{~Hz}$, respectively. The AVM system consists of four cameras at the locations shown in Fig. 12 with red arrows. This system acquires four fisheye images with a resolution of $640 \times 480$ pixels and generates AVM images with a resolution of $360 \times 480$ pixels. Its acquisition frequency is $5 \mathrm{~Hz}$. Wheel speed and steering angle sensors were used for odometry estimation. Odometry contains the orientation angle and displacements in longitudinal and lateral directions with respect to the center of the two rear wheels. The resolutions of the displacement and orientation angle are $0.1 \mathrm{~cm}$ and $0.1^{\circ}$, respectively. Odometry is separately synchronized with an ultrasonic sensor and an AVM image.

Two types of databases were acquired for experiments in practical situations. One is for vacant parking slot marking detection, and the other is for parking slot marking tracking. The first database was taken while the vehicle is moving along corridors of parking lots and includes 102 situations with four types of parking slot markings. This database will be called the "parking slot detection database." The second database was taken while the vehicle was manually moving into parking slots. This database includes 40 situations (ten situations for each type of parking slot marking). This database will be called the "parking slot tracking database." Since the proposed system is not targeted for nighttime and underground parking lots where the quality of the AVM image is not guaranteed due to low illumination conditions, these situations are excluded from our databases.

\section{B. Parking Slot Marking Detection Performance}

This section compares the previous method [31] utilizing single image-based detection and the proposed method utilizing image sequence-based detection. Comparison was conducted using the parking slot detection database composed of 102 situations. Recall and precision in (19) and (20) are utilized as performance measures.

$$
\text { recall }=\frac{\text { No. of correctly detected slots }}{\text { No. of existing slots }}
$$

$$
\text { precision }=\frac{\text { No. of correctly detected slots }}{\text { No. of detected slots }} .
$$

First, the performance of the previous method was evaluated by treating a single AVM image as a test unit. This means that parking slot markings in sequential AVM images are treated as different parking slots even if they are actually images of the same parking slot. Since there are too many individual images in the parking slot detection database, this test was conducted using 992 uniformly sampled AVM images. Table I shows the results of this test. In this evaluation, the previous method gives $59.9 \%$ recall and $90.6 \%$ precision. The reason why the previous method gives low recall is that it fails in 
TABLE I

Performance of the Previous Method With INDIVIDUAL AVM IMAGES

\begin{tabular}{lccccc}
\hline $\begin{array}{c}\text { Slot } \\
\text { type }\end{array}$ & $\begin{array}{c}\text { \# of } \\
\text { existing } \\
\text { slots }\end{array}$ & $\begin{array}{c}\text { \# of } \\
\text { detected } \\
\text { slots }\end{array}$ & $\begin{array}{c}\text { \# of } \\
\text { correctly } \\
\text { detected slots }\end{array}$ & recall & precision \\
\hline TT-slot & 916 & 745 & 716 & $78.2 \%$ & $96.1 \%$ \\
\hline TL-slot & 678 & 376 & 317 & $46.8 \%$ & $84.3 \%$ \\
\hline YY-slot & 779 & 519 & 430 & $55.2 \%$ & $82.9 \%$ \\
\hline II-slot & 927 & 542 & 514 & $55.4 \%$ & $94.8 \%$ \\
\hline Total & $\mathbf{3 3 0 0}$ & $\mathbf{2 1 8 2}$ & $\mathbf{1 9 7 7}$ & $\mathbf{5 9 . 9 \%}$ & $\mathbf{9 0 . 6 \%}$ \\
\hline
\end{tabular}

TABLE II

Performance of the Previous Method With the SUGGESTED CRITERIA

\begin{tabular}{lccccc}
\hline $\begin{array}{c}\text { Slot } \\
\text { type }\end{array}$ & $\begin{array}{c}\text { \# of } \\
\text { existing } \\
\text { slots }\end{array}$ & $\begin{array}{c}\text { \# of } \\
\text { detected } \\
\text { slots }\end{array}$ & $\begin{array}{c}\text { \# of } \\
\text { correctly } \\
\text { detected slots }\end{array}$ & recall & precision \\
\hline TT-slot & 238 & 193 & 90 & $37.8 \%$ & $46.6 \%$ \\
\hline TL-slot & 157 & 277 & 9 & $5.7 \%$ & $3.2 \%$ \\
\hline YY-slot & 198 & 401 & 35 & $17.7 \%$ & $8.7 \%$ \\
\hline II-slot & 223 & 153 & 23 & $10.3 \%$ & $15.0 \%$ \\
\hline Total & $\mathbf{8 1 6}$ & $\mathbf{1 0 2 4}$ & $\mathbf{1 5 7}$ & $\mathbf{1 9 . 2 \%}$ & $\mathbf{1 5 . 3 \%}$ \\
\hline
\end{tabular}

cases where junctions are occluded by adjacent vehicles or degraded at distant locations and stitching lines, as explained in Fig. 3.

The drawbacks of the previous method are more clearly visible when evaluating it by treating an AVM image sequence as a test unit. The proposed system is based on a user interface that displays detected parking slots to a driver while the vehicle is moving along the corridor of the parking lot and the driver selects a desirable one from the detection results. In this user interface, parking slots should be continuously detected through sequential AVM images for drivers to readily select preferable parking slots. Thus, the following evaluation criteria have been suggested. A parking slot is considered to be correctly detected if it is detected before the rear of the vehicle passes by it and the detection holds until it leaves an AVM image or the vehicle stops. A parking slot is considered as a false detection if it is falsely detected once or more through an AVM image sequence.

Table II shows the performance of the previous method with the suggested criteria. In this evaluation, the previous method gives low recall (19.2\%) because it cannot continuously detect parking slots through AVM image sequences due to occlusion and image degradation, as explained in Fig. 3. This low recall makes it difficult for drivers to select desirable parking slots. It also gives low precision (15.3\%) since the suggested criteria treat a single wrongly detected slot as a false detection while treating a sequentially appearing slot as a single slot. Table III
TABLE III

Performance of the Proposed Method With the SUGGESTED CRITERIA

\begin{tabular}{cccccc}
\hline $\begin{array}{c}\text { Slot } \\
\text { type }\end{array}$ & $\begin{array}{c}\text { \# of } \\
\text { existing } \\
\text { slots }\end{array}$ & $\begin{array}{c}\text { \# of } \\
\text { detected } \\
\text { slots }\end{array}$ & $\begin{array}{c}\text { \# of } \\
\text { correctly } \\
\text { detected slots }\end{array}$ & recall & precision \\
\hline TT-slot & 238 & 245 & 234 & $98.3 \%$ & $95.5 \%$ \\
\hline TL-slot & 157 & 163 & 155 & $98.7 \%$ & $95.1 \%$ \\
\hline YY-slot & 198 & 206 & 196 & $99.0 \%$ & $95.1 \%$ \\
\hline II-slot & 223 & 222 & 213 & $95.5 \%$ & $95.9 \%$ \\
\hline Total & $\mathbf{8 1 6}$ & $\mathbf{8 3 6}$ & $\mathbf{7 9 8}$ & $\mathbf{9 7 . 8 \%}$ & $\mathbf{9 5 . 5 \%}$ \\
\hline
\end{tabular}

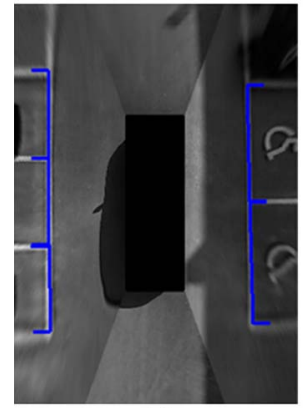

(a)

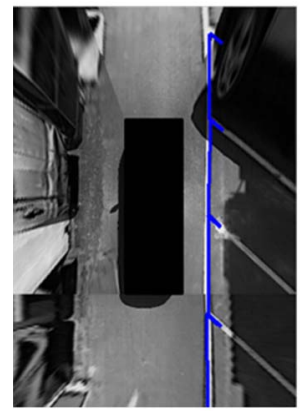

(c)

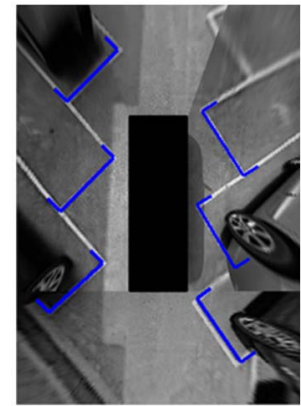

(b)

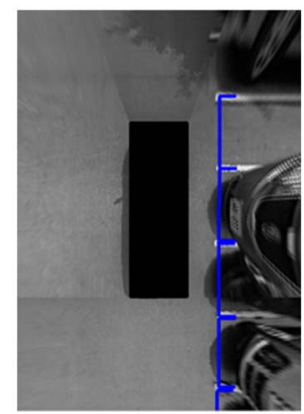

(d)
Fig. 13. Parking slot marking detection results of the proposed method in case of (a) TT-slots, (b) TL-slots, (c) YY-slots, and (d) II-slots.

shows the performance of the proposed parking slot marking detection method with the suggested criteria. The proposed method gives high recall $(97.8 \%)$ because it holds detected slots through sequential AVM images by combining current and previous detection results. This method also gives high precision (95.5\%) since it generates slot candidates using only corners classified as inliers by RANSAC. These inlier corners are more stable than the others because they are consistently detected in consecutive AVM images. In addition, as almost all of the actual slots are correctly detected by exploiting stable corners and sequential detection results, they can naturally suppress falsely detected slots by defeating them during the overlapping slot removal procedure explained in Section III-D. Fig. 13 shows the parking slot marking detection results of the proposed method. It can be seen that this method successfully detects various types of parking slot markings in practical situations. 
TABLE IV

Performance of the Proposed Parking Slot OCCUPANCy ClassificATION METHOD

\begin{tabular}{|c|c|c|c|c|c|c|c|}
\hline $\begin{array}{l}\text { Slot } \\
\text { type }\end{array}$ & 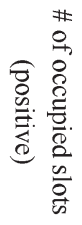 & 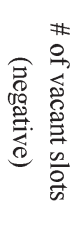 & 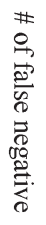 & 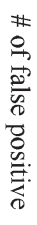 & 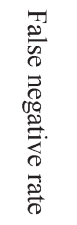 & 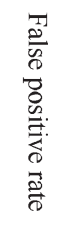 & 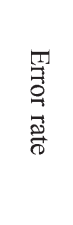 \\
\hline TT-slot & 91 & 143 & 1 & 1 & $1.1 \%$ & $0.7 \%$ & $0.9 \%$ \\
\hline TL-slot & 96 & 59 & 4 & 0 & $4.2 \%$ & $0.0 \%$ & $2.6 \%$ \\
\hline YY-slot & 97 & 99 & 6 & 1 & $6.2 \%$ & $1.0 \%$ & $3.6 \%$ \\
\hline II-slot & 83 & 130 & 2 & 0 & $2.4 \%$ & $0.0 \%$ & $0.9 \%$ \\
\hline Total & 367 & 431 & 13 & 2 & $3.5 \%$ & $0.5 \%$ & $1.9 \%$ \\
\hline
\end{tabular}

\section{Parking Slot Occupancy Classification Performance}

As mentioned in Section IV, to classify occupancies of detected parking slots using (13), $p\left(O \mid z_{t}\right)$, which is either $p(O \mid P)$ or $p(O \mid N)$, should be calculated. $p(P \mid O)$ and $p(P \mid V)$ are estimated with a training database, and then, $p(O \mid P)$ and $p(O \mid N)$ are calculated by Bayes' theorem in

$$
\begin{aligned}
p(O \mid P) & =\frac{p(P \mid O) p(O)}{p(P \mid O) p(O)+p(P \mid V) p(V)} \\
p(O \mid N) & =\frac{\{1-p(P \mid O)\} p(O)}{\{1-p(P \mid O)\} p(O)+\{1-p(P \mid V)\} p(V)} .
\end{aligned}
$$

The training database consists of 813 parking slots of various types with ultrasonic sensor data and includes 456 occupied and 357 vacant parking slots. This database is different from the parking slot detection database. In this database, $p(P \mid O)$ and $p(P \mid V)$ were calculated as 0.795 and 0.056 , respectively. $p(O)$ and $p(V)$ are set to 0.5 by assuming that no prior knowledge about the occupancy status is given. Finally, $p(O \mid P)$ and $p(O \mid N)$ were calculated as 0.934 and 0.178 via (21) and (22), respectively. Based on these probabilities, occupancies of the parking slots in the training database were classified, and the result shows $1.7 \%$ of false positive rate, $1.8 \%$ of false negative rate, and $1.7 \%$ of overall classification error rate.

Table IV shows the parking slot occupancy classification performance with 798 correctly detected parking slots in the parking slot detection database (see Table III). The proposed method gives $3.5 \%$ of false negative rate, $0.5 \%$ of false positive rate, and $1.9 \%$ of the overall classification error rate. The false negative rate of this case is slightly higher than that of the training database case, and most of the false negatives are produced from TL-slots and YY-slots. This is because ultrasonic sensors give unstable outputs in cases of TL-slots and YY-slots where surfaces of parked vehicles are not perpendicular to heading directions of ultrasonic sensors. Fig. 14 shows example results of the proposed parking slot occupancy classification method in various types of parking slot markings. In this figure, white arrows indicate locations and heading directions

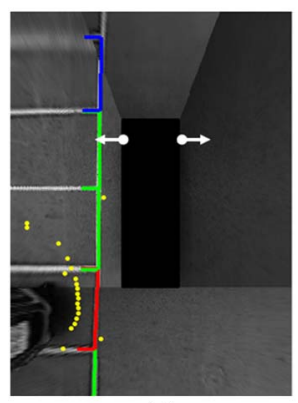

(a)

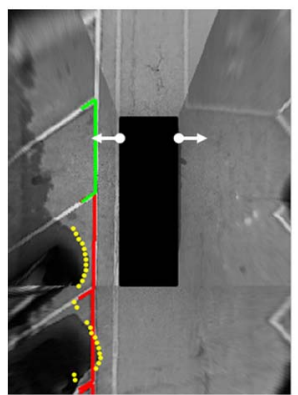

(c)

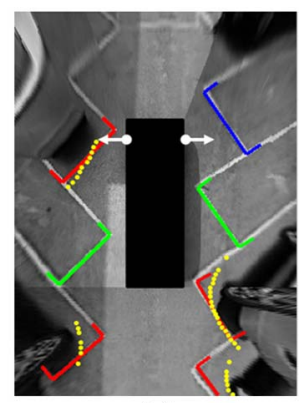

(b)

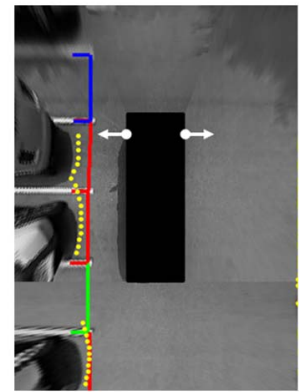

(d)
Fig. 14. Parking slot occupancy classification results of the proposed method in case of (a) TT-slots, (b) TL-slots, (c) YY-slots, and (d) II-slots.

of the ultrasonic sensors, and yellow dots are ultrasonic sensor data. Parking slots with green and red lines indicate vacant and occupied parking slots, respectively, and blue lines are unclassified parking slots where ultrasonic sensors have not yet scanned. It can be noticed that detected parking slots are correctly classified in spite of false positive sensor outputs in the TT-slot in Fig. 14(a) and false-negative sensor outputs in the TL-slot in Fig. 14(b).

\section{Parking Slot Marking Tracking Performance}

This section compares performances of three parking slot marking tracking methods. The first one is an odometry-based method that tracks parking slot markings using motion sensorbased odometry. This method has been widely used in offthe-shelf automatic parking systems [3], [9]-[12]. The second one is an image-based method that uses only AVM images via DCM. The last one is a fusion-based method that fuses the AVM image and odometry information in the DCM score level. These three methods were quantitatively evaluated using the parking slot tracking database that includes 40 practical situations (ten situations for each type of parking slot marking). For quantitative evaluation, we manually designated locations and orientations of junctions that compose tracked parking slots in all AVM images of the parking slot tracking database. Junctions occluded by the ego-vehicle were not designated. Using this ground truth, tracking errors were measured in terms of location and orientation. Location and orientation errors were measured by averaging distances and angles between designated junctions and junctions of the tracked parking slot, respectively. The location error was first measured in pixels and then converted into centimeters using a centimeter-perpixel parameter estimated during AVM system calibration. The orientation error was measured in degrees. 
(a)
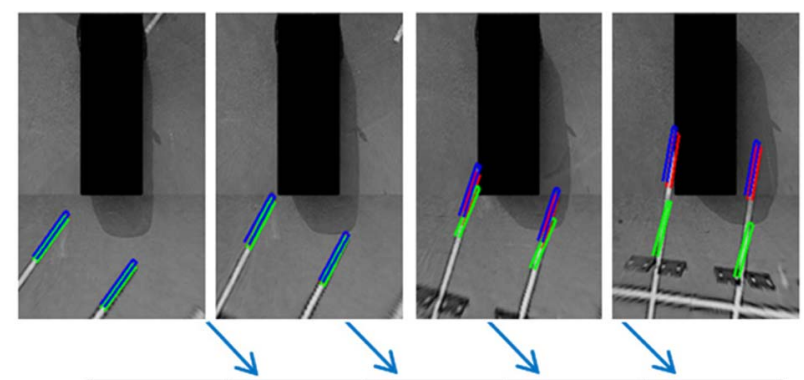

(b)

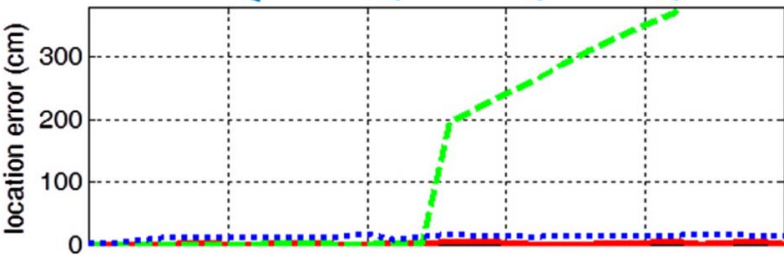

(c)

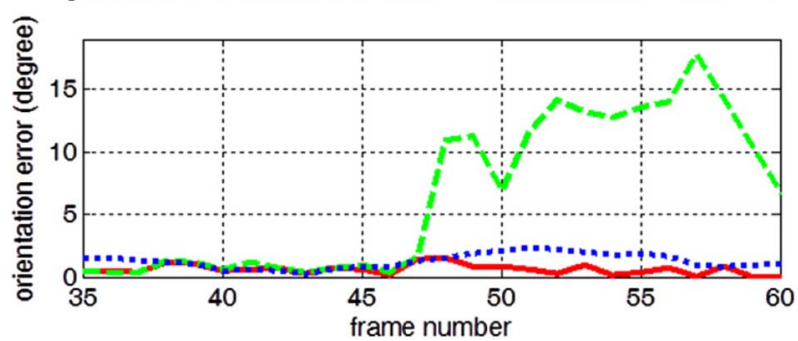

Fig. 15. Location and orientation errors in the case of II-slot tracking.

TABLE V

Mean and Maximum Location Errors of Three Methods (cm)

\begin{tabular}{cccccc}
\hline Method & TT-slot & TL-slot & YY-slot & II-slot & Total \\
\hline $\begin{array}{c}\text { Odometry-based } \\
\text { method }\end{array}$ & $\begin{array}{c}10.4 \\
(25.6)\end{array}$ & $\begin{array}{c}11.6 \\
(27.5)\end{array}$ & $\begin{array}{c}8.4 \\
(22.8)\end{array}$ & $\begin{array}{c}12.2 \\
(36.4)\end{array}$ & $\begin{array}{c}\mathbf{1 0 . 8} \\
\mathbf{( 3 6 . 4 )}\end{array}$ \\
\hline $\begin{array}{c}\text { Image-based } \\
\text { method }\end{array}$ & $\begin{array}{c}27.7 \\
(255.9)\end{array}$ & $\begin{array}{c}3.0 \\
(8.3)\end{array}$ & $\begin{array}{c}4.0 \\
(11.4)\end{array}$ & $\begin{array}{c}21.7 \\
(444.4)\end{array}$ & $\mathbf{1 4 . 9}$ \\
\hline $\begin{array}{c}\mathbf{4 4 4 . 4}) \\
\text { Fusion-based } \\
\text { method }\end{array}$ & $\begin{array}{c}3.0 \\
(9.4)\end{array}$ & $\begin{array}{c}3.2 \\
(8.0)\end{array}$ & $\begin{array}{c}3.9 \\
(10.3)\end{array}$ & $\begin{array}{c}2.4 \\
(9.8)\end{array}$ & $\mathbf{3 . 1}$ \\
\hline & & & & & \\
\hline
\end{tabular}

Fig. 15 shows the location and orientation errors in the case of II-slot tracking. This is the same situation as Figs. 9 and 11. In Fig. 15(a), blue, green, and red lines are tracking results of odometry-based, image-based, and fusion-based methods, respectively. Blue dotted, green dashed, and red solid lines in Fig. 15(b) and (c) indicate location and orientation errors of odometry-based, image-based, and fusion-based methods, respectively. As mentioned in Section V, the tracking results of the odometry-based method are stable but more erroneous than the proposed fusion-based method due to the cumulative error of odometry. Tracking results of the image-based method are less erroneous in the case of moderate occlusions, but it becomes unstable when severe occlusions occur on important parts of the parking slot marking. However, the proposed fusion-based method gives accurate and stable tracking results regardless of severe occlusions or odometry error accumulation.

Tables V and VI summarize location and orientation errors of three methods in cases of four types of parking slots, respectively. In these tables, numbers with and without parenthesis
TABLE VI

MEAN AND MAXIMUM ORIENTATION ERRORS OF Three Methods (Degrees)

\begin{tabular}{cccccc}
\hline Method & TT-slot & TL-slot & YY-slot & II-slot & Total \\
\hline $\begin{array}{c}\text { Odometry-based } \\
\text { method }\end{array}$ & $\begin{array}{c}1.2 \\
(4.2)\end{array}$ & $\begin{array}{c}2.5 \\
(6.0)\end{array}$ & $\begin{array}{c}1.2 \\
(4.2)\end{array}$ & $\begin{array}{c}1.4 \\
(4.8)\end{array}$ & $\begin{array}{c}\mathbf{1 . 6} \\
\mathbf{( 6 . 0 )}\end{array}$ \\
\hline $\begin{array}{c}\text { Image-based } \\
\text { method }\end{array}$ & $\begin{array}{c}1.0 \\
(6.3)\end{array}$ & $\begin{array}{c}1.2 \\
(4.5)\end{array}$ & $\begin{array}{c}0.9 \\
(3.0)\end{array}$ & $\begin{array}{c}0.9 \\
(17.8)\end{array}$ & $\mathbf{1 . 0}$ \\
\hline $\begin{array}{c}\mathbf{1 7 . 8}) \\
\text { Fusion-based } \\
\text { method }\end{array}$ & $\begin{array}{c}0.9 \\
(3.4)\end{array}$ & $\begin{array}{c}1.2 \\
(3.8)\end{array}$ & $\begin{array}{c}0.9 \\
(2.5)\end{array}$ & $\begin{array}{c}0.7 \\
(3.0)\end{array}$ & $\mathbf{0 . 9}$ \\
\hline
\end{tabular}

indicate mean and maximum errors, respectively. As a result, the proposed fusion-based method gives an average of $3.1 \mathrm{~cm}$ and $0.9^{\circ}$ errors and a maximum of $10.3 \mathrm{~cm}$ and $3.8^{\circ}$ errors in terms of location and orientation, respectively. This reveals that the proposed fusion-based method outperforms the odometrybased method by approximately 3.5 and 1.8 times in terms of location and orientation errors, respectively. The image-based method gives quite large maximum errors in cases of TT-slot and II-slot due to failures caused by severe occlusions. Fig. 16 shows example results of the proposed fusion-based method in sequential AVM images. In Fig. 16(a), the proposed method properly tracks a TT-slot in cases where a parking slot marking is occluded by both the ego-vehicle and two adjacent vehicles. In Fig. 16(b), a TL-slot is correctly tracked although some of its parts are out of AVM images at the initial frames. In Fig. 16(c), a YY-slot is successfully tracked even in case where the parking slot marking is partially covered with snow. From these results, it can be expected that the proposed method enhances the accuracy of parking control by continuously updating the relative position between the ego-vehicle and the desirable parking slot.

\section{E. Execution Time}

All methods were implemented in $\mathrm{C}$ language, and their execution times were measured on a $3.40-\mathrm{GHz}$ Intel Core i72600 CPU with 4-G RAM. Parking slot marking detection and occupancy classification stages require $47.1 \mathrm{~ms}$ per frame. Almost all computation time is consumed by the parking slot marking detection stage, and the occupancy classification stage consumes much less than $0.1 \mathrm{~ms}$ per frame. The proposed fusion-based parking slot marking tracking method requires $32.0 \mathrm{~ms}$ per frame. For reference, the image-based tracking method requires $82.1 \mathrm{~ms}$ per frame, which is mainly due to large search ranges. These results reveal that the proposed system can surely operate in real time. It is notable that the parking slot marking detection and occupancy classification stages simultaneously operate and that the parking slot marking tracking stage operates alone after the two former stages.

\section{F. Discussions and Future Works}

This paper proposes a system that detects and tracks parking slot markings in daytime situations by fusing the sensors of an AVM system and an ultrasonic sensor-based automatic parking system. Since the proposed system provides vacant parking 


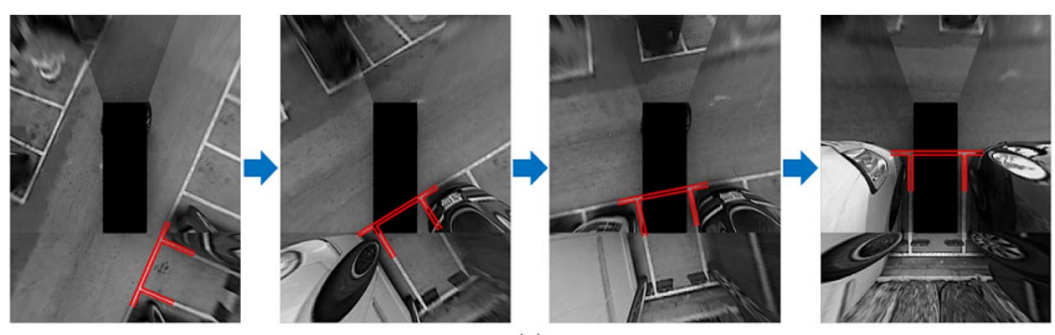

(a)
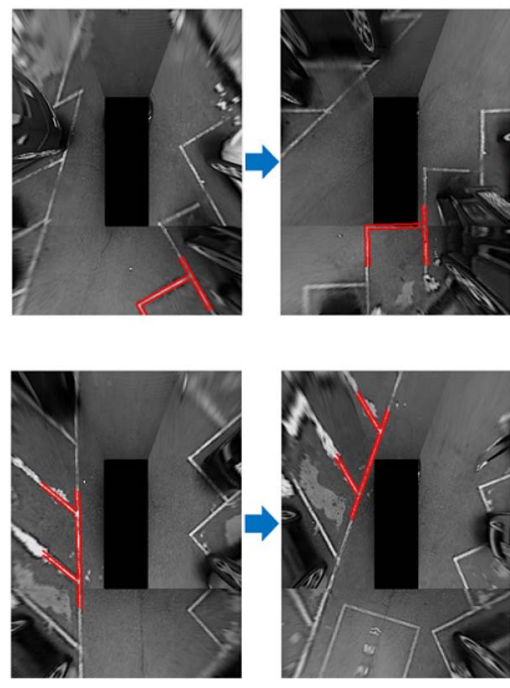

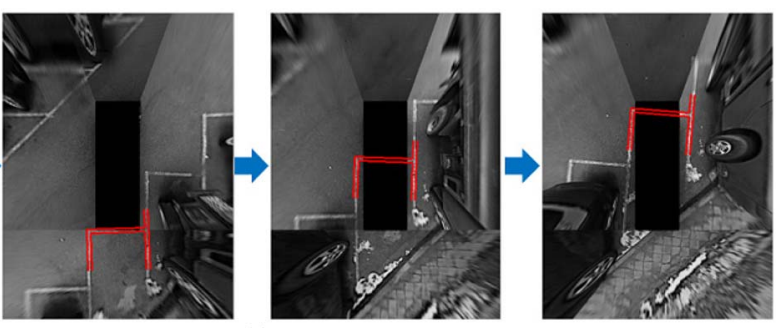

(b)

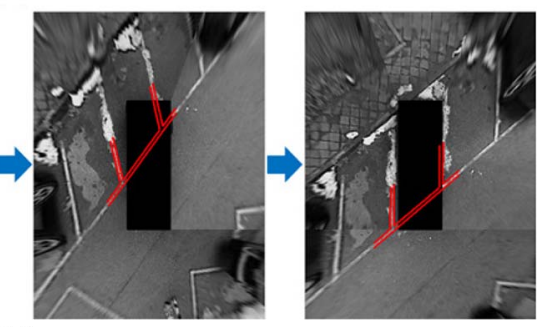

(c)

Fig. 16. Example results of the proposed fusion-based method in case of (a) TT-slot, (b) TL-slot, and (c) YY-slot.

slots, drivers only need to select one of the provided slots. Considering that drivers should use a small-sized touch screen sitting on a cramped and uncomfortable car seat, the proposed system is more convenient than the previous systems that ask drivers to point out relatively precise locations of one or two junctions [26], [27]. This system can also successfully detect YY-slots and TL-slots that are unable to be handled by ultrasonic sensor-based automatic parking systems because vehicle surfaces are usually not perpendicular to heading directions of ultrasonic sensors in these cases. Furthermore, the proposed system makes it possible for the relative position between the ego-vehicle and the selected parking slot to be continuously updated by tracking its positions in sequential AVM images. This procedure is expected to reduce 3-D parking slot position errors caused by nonflat road surfaces and slight changes in camera positions and induce accurate vehicle control into the parking slot.

However, the proposed system does not properly work in cases of nighttime and underground parking lots and severely damaged parking slot markings since it basically utilizes outputs of vision sensors. It would be best to handle all these situations by a single method; however, it is practically difficult and inefficient in terms of algorithm development and performance achievement. Thus, we are currently developing a method that is specialized for underground parking lots where dim lighting, reflection, and noise multiplication are presented. After that, parking situations are classified into three types, i.e., outdoor daytime, outdoor nighttime, and underground, and a different approach will be applied to each situation. The system proposed in this paper will handle outdoor daytime situations, a conventional ultrasonic sensor-based automatic parking system will deal with outdoor nighttime situations, and the method currently being developed will cope with underground situations. In addition, we are planning to use cameras with high dynamic ranges for nighttime and underground situations and develop a method that can figure out if recognizable parking slot markings are presented. Situations without recognizable parking slot markings will be handled by a conventional ultrasonic sensorbased automatic parking system.

\section{CONCLUSION}

This paper has proposed a vacant parking slot detection and tracking system that fuses the sensors of an AVM system and an ultrasonic sensor-based automatic parking system. This paper has presented that 1) parking slot markings can be successfully detected and tracked by fusing two off-the-shelf parking aid systems, 2) parking slot markings can be reliably detected in AVM image sequences by combining sequential detection results, 3) occupancy of parking slot can be efficiently classified by treating each parking slot region as a cell of an occupancy grid, and 4) parking slot markings can be tracked robust against severe occlusions by fusing an AVM image and odometry in the DCM score level. As a result of quantitative evaluation using practical databases, the proposed system achieves $97.8 \%$ recall and $95.8 \%$ precision for parking slot marking detection, $98.1 \%$ classification rate for parking slot occupancy classification, and accuracy of $3.1 \mathrm{~cm}$ and $0.9^{\circ}$ for parking slot marking tracking. 
Furthermore, it is revealed that the proposed system can operate in real time, i.e., $47.1 \mathrm{~ms}$ for parking slot marking detection and occupancy classification and $32.0 \mathrm{~ms}$ for parking slot marking tracking.

\section{REFERENCES}

[1] S. Hiramatsu, A. Hibi, Y. Tanaka, T. Kakinami, Y. Iwata, and M. Nakamura, "Rearview camera based parking assist system with voice guidance," presented at the Proc. SAE World Congr. Exhib., Detroit, MI, USA, Apr. 2002, Paper 2002-01-0759.

[2] M. Furutani, "Obstacle detection systems for vehicle safety," presented at the Proc. SAE Converg. Int. Congr. Expo. Transp. Electron., Detroit, MI, USA, Oct. 2004, Paper 2004-21-0057.

[3] Y. Kageyama, Look, No Hand! New Toyota Parks Itself, Jan. 14, 2004. [Online]. Available: http://www.cnn.com/

[4] H. G. Jung, C. G. Choi, P. J. Yoon, and J. Kim, "Novel user interface for semi-automatic parking assistance system," in Proc. 31st FISITA World Autom. Congr., Oct. 2006, pp. 1-10.

[5] H. Satonaka, M. Okuda, S. Hayasaka, T. Endo, Y. Tanaka, and T. Yoshida, "Development of parking space detection using an ultrasonic sensor," in Proc. 13th World Congr. Intell. Transp. Syst. Serv., Oct. 2006, pp. 1-10.

[6] P. Degerman, J. Pohl, and M. Sethson, "Hough transform for parking space estimation using long range ultrasonic sensors," presented at the Proc. SAE World Congr. Exhib., Detroit, MI, USA, Apr. 2006, Paper 2006-01-0810.

[7] W. J. Park, B. S. Kim, D. E. Seo, D. S. Kim, and K. H. Lee, "Parking space detection using ultrasonic sensor in parking assistance system," in Proc. IEEE Intell. Veh. Symp., Jun. 2008, pp. 1039-1044.

[8] S. H. Jeong, C. G. Choi, J. N. Oh, P. J. Yoon, B. S. Kim, M. Kim, and K. H. Lee, "Low cost design of parallel parking assist system based on an ultrasonic sensor," Int. J. Autom. Technol., vol. 11, no. 3, pp. 409-416, Jun. 2010.

[9] Ford 2013 TAURUS. [Accessed: Mar. 2013]. [Online]. Available: http:// www.ford.com/cars/taurus/features/\#page $=$ Feature 18

[10] BMW 7 Series Sedan. [Accessed: Mar. 2013]. [Online]. Available: http:// www.bmw.com/com/en/newvehicles/7series/sedan/2012/showroom/ convenience/park-assistant.html

[11] Lexus 2013 LS. [Accessed: Mar. 2013]. [Online]. Available: http://www. lexus.com/models/LS/features/

[12] Hyundai 2013 AZERA (GRANDEUR). [Accessed: Mar. 2013]. [Online]. Available: http://www.hyundai.com/kr/showroom.do?carCd1=RD014

[13] A. Hashizume, S. Ozawa, and H. Yanagawa, "An approach to detect vacant parking space in a parallel parking area," in Proc. 5th Eur. Congr. Exhib. Intell. Transp. Syst. Serv., Jun. 2005, pp. 1-5.

[14] C. Vestri, S. Bougnoux, R. Bendahan, K. Fintzel, S. Wybo, F. Abad, and T. Kakinami, "Evaluation of a vision-based parking assistance system," in Proc. 8th Int. IEEE Conf. Intell. Transp. Syst., Sep. 2005, pp. 131-135.

[15] J. K. Suhr, H. G. Jung, K. Bae, and J. Kim, "Automatic free parking space detection by using motion stereo-based 3D reconstruction," Mach. Vis. Appl., vol. 21, no. 2, pp. 163-176, Feb. 2010.

[16] C. Unger, E. Wahl, and S. Ilic, "Parking assistance using dense motionstereo: Real-time parking slot detection, collision warning and augmented parking," Mach. Vis. Appl., to be published.

[17] N. Kaempchen, U. Franke, and R. Ott, "Stereo vision based pose estimation of parking lots using 3-D vehicle models," in Proc. IEEE Intell. Veh. Symp., Jun. 2002, pp. 459-464.

[18] H. G. Jung, D. S. Kim, P. J. Yoon, and J. H. Kim, "3D vision system for the recognition of free parking site location," Int. J. Autom. Technol., vol. 7, no. 3, pp. 361-367, May 2006.

[19] H. G. Jung, D. S. Kim, and J. Kim, "Light stripe projection-based target position designation for intelligent parking-assist system," IEEE Trans. Intell. Transp. Syst., vol. 11, no. 4, pp. 942-953, Dec. 2010.

[20] F. Abad, R. Bendahan, S. Wybo1, S. Bougnoux, C. Vestri, and T. Kakinami, "Parking space detection," in Proc. 14th World Congr. Intell. Transp. Syst., Oct. 2007, pp. 1-8.

[21] H. G. Jung, Y. H. Cho, P. J. Yoon, and J. Kim, "Scanning laser radarbased target position designation for parking aid system," IEEE Trans. Intell. Transp. Syst., vol. 9, no. 3, pp. 406-424, Sep. 2008.

[22] J. Zhou, L. E. Navarro-Serment, and M. Hebert, "Detection of parking spots using 2D range data," in Proc. 15th Int. IEEE Conf. Intell. Transp. Syst., Sep. 2012, pp. 1280-1287.

[23] S. Görner and H. Rohling, "Parking lot detection with $24 \mathrm{GHz}$ radar sensor," in Proc. 3rd Int. Workshop Intell. Transp., Mar. 2006, pp. 1-6.
[24] M. R. Schmid, S. Ates, J. Dickmann, F. Hundelshausen, and H. J. Wuensche, "Parking space detection with hierarchical dynamic occupancy grids," in Proc. IEEE Intell. Veh. Symp., Jun. 2011, pp. 254-259.

[25] U. Scheunert, B. Fardi, N. Mattern, G. Wanielik, and N. Keppeler, "Free space determination for parking slots using a 3D PMD sensor," in Proc. IEEE Intell. Veh. Symp., Jun. 2007, pp. 154-159.

[26] H. G. Jung, D. S. Kim, P. J. Yoon, and J. Kim, "Structure analysis based parking slot marking recognition for semi-automatic parking system," in Proc. Lect. Notes Comput. Sci., Aug. 2006, vol. 4109, pp. 384-393.

[27] H. G. Jung, Y. H. Lee, and J. Kim, "Uniform user interface for semiautomatic parking slot marking recognition," IEEE Trans. Veh. Technol., vol. 59, no. 2, pp. 616-626, Feb. 2010.

[28] J. Xu, G. Chen, and M. Xie, "Vision-guided automatic parking for smart car," in Proc. IEEE Intell. Veh. Symp., Oct. 2000, pp. 725-730.

[29] H. G. Jung, D. S. Kim, P. J. Yoon, and J. Kim, "Parking slot markings recognition for automatic parking assist system," in Proc. IEEE Intell. Veh. Symp., Jun. 2006, pp. 106-113.

[30] Y. Tanaka, M. Saiki, M. Katoh, and T. Endo, "Development of image recognition for a parking assist system," in Proc. 14th World Congr. Intell. Transp. Syst. Serv., Oct. 2006, pp. 1-7.

[31] J. K. Suhr and H. G. Jung, "Full-automatic recognition of various parking slot markings using a hierarchical tree structure," Opt. Eng., vol. 52, no. 3, pp. 037203-1-037203-14, Mar. 2013.

[32] M. Y. I. Idris, Y. Y. Leng, E. M. Tamil, N. M. Noor, and Z. Razk, "Car park system: A review of smart parking system and its technology," Inf. Technol. J., vol. 8, no. 2, pp. 101-113, 2009.

[33] G. Yan, W. Yang, D. B. Rawat, and S. Olariu, "SmartParking: A secure and intelligent parking system," IEEE Trans. Intell. Transp. Syst. Mag., vol. 3, no. 1, pp. 18-30, Spring 2011.

[34] Y. Suzuki, M. Koyamaishi, T. Yendo, T. Fujii, and M. Tanimoto, "Parking assistance using multi-camera infrastructure," in Proc. IEEE Intell. Veh. Symp., Jun. 2005, pp. 106-111.

[35] M. Wada, K. S. Yoon, and H. Hashimoto, "Development of advanced parking assistance system," IEEE Trans. Ind. Electron., vol. 50, no. 1, pp. 4-17, Feb. 2003.

[36] M. Wada, X. Mao, H. Hashimoto, M. Mizutani, and M. Saito, "iCAN Pursuing technology for near-future ITS," IEEE Intell. Syst., vol. 19, no. 1, pp. 18-23, Jan./Feb. 2004

[37] K. An, J. Choi, and D. Kwak, "Automatic valet parking system Incorporating a Nomadic device and parking servers," in Proc. IEEE Int. Conf. Consum. Electron., Jan. 2011, pp. 111-112.

[38] K. Sung, J. Choi, and D. Kwak, "Vehicle control system for automatic valet parking with infrastructure sensors," in Proc. IEEE Int. Conf. Consum. Electron., Jan. 2011, pp. 567-568.

[39] V-Charge: Autonomous Valet Parking and Charging for e-Mobility. [Accessed: Mar. 2013]. [Online]. Available: http://www.v-charge.eu/

[40] Nissan-Around View Monitor System. [Accessed: Mar. 2013]. [Online]. Available: http://www.nissan-global.com/EN/TECHNOLOGY/ OVERVIEW/avm.html

[41] Honda-Multi-View Camera System. [Accessed: Mar. 2013]. [Online]. Available: http://world.honda.com/news/2008/4080918Multi-ViewCamera-System

[42] BMW-Surround View System. [Accessed: Mar. 2013]. [Online]. Available: http://www.bmw.com/com/en/insights/technology/connecteddrive/ 2010/convenience/parking/surround_view_information.html

[43] Hyundai Mobis- Around View Monitoring System. [Accessed: Mar. 2013]. [Online]. Available: http://www.mobis.co.kr/Eng/PR/News/View. aspx?idx=219

[44] J. K. Suhr and H. G. Jung, "Fully-automatic recognition of various parking slot markings in Around View Monitor (AVM) image sequences," in Proc. 15th Int. IEEE Conf. Intell. Transp. Syst., Sep. 2012, pp. 1294-1299.

[45] C. Harris and M. Stephens, "A combined corner and edge detector," in Proc. 4th Alvey Vis. Conf., Sep. 1988, pp. 147-151.

[46] R. Hartley and A. Zisserman, Multiple View Geometry in Computer Vision, 2nd ed. Cambridge, U.K.: Cambridge Univ. Press, 2003.

[47] J. C. Gower and G. B. Dijksterhuis, Procrustes Problems. New York, NY, USA: Oxford Univ. Press, 2004.

[48] R. C. Gonzalez and R. E. Woods, Digital Image Processing, 3rd ed. Englewood Cliffs, NJ, USA: Prentice-Hall, 2008.

[49] M. Fischler and R. Bolles, "Random sample consensus: A paradigm for model fitting with applications to image analysis and automated cartography," Commun. ACM, vol. 24, no. 6, pp. 381-395, Jun. 1981.

[50] R. J. Radke, S. Andra, O. Al-Kofahi, and B. Roysam, "Image change detection algorithms: A systematic survey," IEEE Trans. Image Process. vol. 14 , no. 3, pp. 294-307, Mar. 2005. 
[51] A. K. Jain, K. Nandakumar, and A. Ross, "Score normalization in multimodal biometric systems," Pattern Recognit., vol. 38, no. 12, pp. 2270-2285, Dec. 2005.

[52] S. Thrun, W. Burgard, and D. Fox, Probabilistic Robotics. Cambridge, MA, USA: MIT Press, 2005.

[53] B. Stenger, A. Thayananthan, P. H. S. Torr, and R. Cipolla, "Model-based hand tracking using a hierarchical bayesian filter," IEEE Trans. Pattern Anal. Mach. Intell., vol. 28, no. 9, pp. 1372-1384, Sep. 2006.

[54] R. Rajamani, Vehicle Dynamics and Control, 2nd ed. New York, NY, USA: Springer-Verlag, 2012.

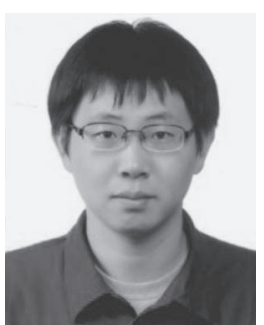

Jae Kyu Suhr (M'12) received the B.S. degree in electronic engineering from Inha University, Incheon, Korea, in 2005 and the M.S. and Ph.D. degrees in electrical and electronic engineering from Yonsei University, Seoul, Korea, in 2007 and 2011, respectively.

$\mathrm{He}$ is currently a Postdoctoral Researcher with the Research Institute of Automotive Electronics and Control, Hanyang University, Seoul. His current research interests include computer vision, image analysis, and pattern recognition for intelligent vehicle and visual surveillance.

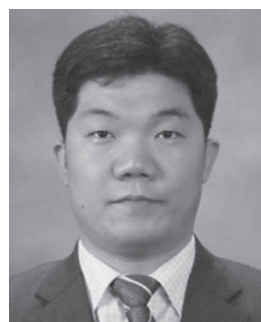

Ho Gi Jung (M'05-SM'10) received the B.E., M.E., and $\mathrm{Ph} . \mathrm{D}$. degrees in electronic engineering from Yonsei University, Seoul, Korea, in 1995, 1997, and 2008, respectively.

From 1997 to April 2009, he was with MANDO Corporation Global R\&D H.Q., where he developed environmental recognition systems for an intelligent parking assist system, a collision warning and avoidance system, and an active pedestrian protection system. From May 2009 to February 2011, he was a Full-Time Researcher and a Research Professor with Yonsei University, working on computer vision applications for intelligent surveillance systems and biometric systems. Since March 2011, he has been an Assistant Professor with Hanyang University, Seoul, working on recognition systems for intelligent vehicles. His interests include recognition systems for intelligent vehicles, next-generation vehicles, computer vision applications, and pattern recognition applications. 